\title{
Enduring Changes in Neuronal Function upon Systemic Inflammation Are NLRP3 Inflammasome Dependent
}

\author{
Marianna M.S. Beyer, ${ }^{1,2 \star}$ Niklas Lonnemann, ${ }^{1,2 \star}$ Anita Remus, ${ }^{1,2}$ Eicke Latz, ${ }^{3,4}$ Michael T. Heneka, ${ }^{4,5}$ and \\ (iD) Martin Korte ${ }^{1,2}$ \\ ${ }^{1}$ Division of Cellular Neurobiology, Zoological Institute, Technische Universität Braunschweig, 38106 Braunschweig, Germany, ${ }^{2}$ Research Group \\ Neuroinflammation and Neurodegeneration, Helmholtz Centre for Infection Research, 38124 Braunschweig, Germany, ${ }^{3}$ German Center for \\ Neurodegenerative Disease (DZNE), 53127 Bonn, Germany, ${ }^{4}$ Institute of Innate Immunity, University of Bonn, Bonn 53127, Germany, and \\ ${ }^{5}$ Department of Neurodegenerative Disorders and Gerontopsychiatry, University of Bonn, Bonn 53127, Germany
}

Neuroinflammation can be caused by various insults to the brain and represents an important pathologic hallmark of neurodegenerative diseases including Alzheimer's disease (AD). Infection-triggered acute systemic inflammation is able to induce neuroinflammation and may negatively affect neuronal morphology, synaptic plasticity, and cognitive function. In contrast to acute effects, persisting consequences for the brain on systemic immune stimulation remain largely unexplored. Here, we report an age-dependent vulnerability of wild-type (WT) mice of either sex toward a systemic immune stimulation by Salmonella typhimurium lipopolysaccharide (LPS). Decreased neuronal complexity three months after peripheral immune stimulation is accompanied by impairment in long-term potentiation (LTP) and spatial learning. Aged APP/PS1 mice reveal an increased sensitivity also to LPS of Escherichia coli, which had no effect in WT mice. We further report that these effects are mediated by NOD-like receptor family, pyrin domain containing 3 (NLRP3) inflammasome activation, since the genetic ablation and pharmacological inhibition using the NLRP3 inhibitor MCC950 rescue the morphological and electrophysiological phenotype.

Key words: APP/PS1; hippocampus; LPS; neuroinflammation; NLRP3; sepsis

Significance Statement

Acute peripheral immune stimulation has been shown to have both positive and negative effects on $\mathrm{A} \beta$ deposition. Improvements or worsening may be possible in acute inflammation. However, there is still no evidence of effects longer than a month after stimulation. The data are pointing to an important role of the NOD-like receptor family, pyrin domain containing 3 (NLRP3) inflammasome for mediating the long-term consequences of systemic immune stimulation, which in addition turns out to be age dependent.

\section{Introduction}

Neuroinflammation and activation of brain resident cells involved in innate immunity are associated with injury, infection, neurodegeneration or autoimmune disease. Here, infection of the brain parenchyma as well as the periphery such as severe

Received Jan. 24, 2020; revised May 25, 2020; accepted May 28, 2020.

Author contributions: M.T.H. and M.K. designed research; M.M.S.B. and N.L. performed research; E.L. contributed unpublished reagents/analytic tools; M.M.S.B., N.L., A.R., and M.K. analyzed data; M.T.H. and M.K. wrote the paper.

${ }^{*}$ M.M.S.B. and N.L. contributed equally to this work.

This work was in part supported by JNPD/INCURE: EU Joint Programme - Neurodegenerative Disease Research (JPND) (BMBF) (M.T.H. and M.K.), the Niedersachsen-Research Network on Neuroinfectiology (NRENNT) of the Ministry of Science and Culture of Lower Saxony (M.K.), and the DFG Grant SFB854 (to M.K.).

The authors declare no competing financial interests.

Correspondence should be addressed to Martin Korte at m.korte@tu-bs.de or Michael T. Heneka at michael.heneka@ukb.uni-bonn.de.

https://doi.org/10.1523/JNEUROSCI.0200-20.2020

Copyright $\odot 2020$ the authors bacteremia and sepsis can trigger neuroinflammation. Sepsis and septic shock as systemic inflammatory response to infection with bacteria, viruses or fungi exhibit a high lethality and are among the leading causes of death in intensive care units worldwide (Bone, 1992; Angus et al., 2001). Severe acute infection causes inflammation-induced encephalopathy and leads to impaired attention (Pine et al., 1983; Sprung et al., 1990; Wilson and Young, 2003).

Intraperitoneal administration of lipopolysaccharide (LPS) from various bacterial sources is widely used to model infection and sepsis in mice. Studies show that the application of this endotoxic Gram-negative bacterial cell wall component is sufficient to induce high production of IL- $1 \beta$ and TNF $\alpha$ (Michie et al., 1988; Cannon et al., 1990). The acute consequences of such a peripheral LPS challenge range from impairment in long-term potentiation (LTP; Commins et al., 2001; Hennigan et al., 2007; Di Filippo et al., 2013; Strehl et al., 2014) to deficits in learning and memory (Arai et al., 2001; Shaw et al., 2001; Sparkman et al., 
2005). In LPS-stimulated mice, the level of blood or liver cytokines increases only hours after administration, but also the cytokine production in the brain parenchyma is elevated since microglia, the resident macrophages and primary source of cytokines in the brain, react to peripheral inflammatory signals (Nguyen et al., 2002). Microglia are activated transiently by inflammatory stimuli and return to their physiological state and surveillance function once infection, injury or peripheral inflammation has been overcome. This mechanism, however, might be disturbed in the elderly as many markers of classical microglia activation are upregulated in mice and also humans as consequence of normal aging (Godbout et al., 2005; Letiembre et al., 2007; Norden and Godbout, 2013).

Increasing evidence suggests a connection between inflammatory conditions in the brain/body and Alzheimer's disease (AD; Holmes et al., 2009; Heneka et al., 2015). For example, A $\beta$ aggregation and senile plaque formation was found to be enhanced due to the production of nitric oxide as inflammatory response and the APP/PS1 phenotype was rescued by depletion of the NOD-like receptor family, pyrin domain containing 3 (NLRP3) inflammasome responsible for IL-1 $\beta$ and IL-18 maturation (Kummer et al., 2011; Heneka et al., 2013). NLRP3 expression is activated in parallel to pro-IL-1 $\beta$ and pro-IL-18 expression usually by pathogen associated molecular patterns like LPS but also by amyloid- $\beta$ aggregates (Halle et al., 2008; Heneka et al., 2013). By now, the NLRP3 inflammasome has been implicated in several systemic inflammatory diseases and in various neurologic disorders (Latz, 2010; Heneka et al., 2018).

Several studies suggest worsening of $\mathrm{A} \beta$ accumulation, tau pathology and cognitive function in murine $\mathrm{AD}$ models on peripheral immune stimulation with LPS (Qiao et al., 2001; Sheng et al., 2003; Lee et al., 2010; Valero et al., 2014), while others report an enhanced $\mathrm{A} \beta$ clearance in response to proinflammatory conditions (DiCarlo et al., 2001; Michaud et al., 2013). Systemic inflammation, has clinically been shown, leads to persisting neurocognitive changes (Semmler et al., 2013) and increases the risk for the subsequent development of dementia (Iwashyna et al., 2010; Holmes and Butchart, 2011).

In this study, we report a neurodegenerative phenotype in 19month-old wild-type (WT) mice three months after peripheral immune challenge with LPS of Salmonella typhimurium. This time point has been chosen to specifically address an infection challenge of an aged population, as the mouse model starts to deposit $\mathrm{A} \beta$ by six months, with the strongest increase in $\mathrm{A} \beta$ deposition over the following six months (Jankowsky et al., 2004b). This phenotype is promoted by the NLRP3 inflammasome as the NLRP3 knock-out (KO) model and WT mice treated with the NLRP3 inflammasome inhibitor MCC950 (Coll et al., 2015) display normal neuronal morphology and function. Nineteenmonth-old APP/PS1 mice on the other hand are more susceptible even to Escherichia coli LPS which had no effect in WT mice.

\section{Materials and Methods}

\section{Animals}

All animals used for this study were of either sex and housed under standard conditions in a $12 / 12 \mathrm{~h} \mathrm{light} /$ dark cycle at $22.0^{\circ} \mathrm{C}$ with food and water available ad libitum. All experiments performed were authorized by the animal welfare representative of the Technische Universität Braunschweig and the LAVES [Oldenburg, Germany, Az. $\$ 4$ (02.05) TSchB TU BS]. The AD mouse model used in this study was the B6C3-Tg (APPswe K595N/M596L, PSEN1dE9) 85Dbo/Mmjax on C57BL/6J background provided by David Borchelt (McKnight Brain Institute, University of Florida). NLRP3-deficient animals (Millennium Pharmaceuticals) were backcrossed to C57BL/6J mice to $>99 \%$ C57BL/6, as confirmed by microsatellite analysis.

\section{LPS administration}

For the systemic immune stimulation with LPS, the body weight of the young adult (four months) and aged (13-16 months) WT, APP/PS1, and NLRP3 KO mice was monitored for one week before the experiment, and the appropriate volume of LPS from E. coli (O127:B8, SigmaAldrich L 3129), LPS from Salmonella enterica serogroup typhimurium (Sigma-Aldrich L 6511), or $0.9 \%$ sodium chloride was calculated for each mouse. On two consecutive days, $0.2 \mu \mathrm{g} / \mathrm{g}$ bodyweight LPS diluted in $0.9 \% \mathrm{NaCl}$ was given intraperitoneally. The same volume of $0.9 \%$ $\mathrm{NaCl}$ was injected as control. Body weight loss and sickness behavior were controlled for $14 \mathrm{~d}$ after immune stimulation.

To determine the effect of low dose LPS administration on cytokine expression, $0.2 \mu \mathrm{g} / \mathrm{g}$ bodyweight LPS was injected in 13-month-old WT mice. Blood serum levels and brain tissue were analyzed $6 \mathrm{~h}$ after LPS stimulus.

\section{Endotoxin quantitation}

LPS from E. coli and S. typhimurium were tested for their endotoxin levels with the limulus amebocyte lysate (LAL) assay (Pierce LAL Chromogenic Endotoxin Quantitation kit) as indicated by the manufacturer.

The Pierce LAL Chromogenic Endotoxin Quantitation kit (Thermo Scientific) measures the levels of endotoxins in a protein sample using the LAL test. The test is very sensitive and can detect levels starting with $0.01 \mathrm{ng}$ endotoxin $/ \mathrm{ml}$. Applied protocol: (1) endotoxin standard stock solution $(1.00,0.50,0.25,0.10 \mathrm{EU} / \mathrm{ml})$ in endotoxin-free water; (2) reconstitute LAL reagent in endotoxin-free water; (3) reconstitute the chromogenic substrate in endotoxin-free water; (4) assay procedure: (1) pre-equilibrate the microplate in a heating block for $10 \mathrm{~min}$ at $37^{\circ} \mathrm{C}$; (2) maintaining microplate at $37^{\circ} \mathrm{C}$, carefully dispense $50 \mu \mathrm{l}$ of each standard and unknown sample; (3) at time $\mathrm{T}=0$ add $50 \mu \mathrm{l}$ of LAL reagent; begin timing as the LAL is added; once LAL is added to each well, briefly remove from heating block and gently tap several times; incubate at $37^{\circ}$ $\mathrm{C}$ for $10 \mathrm{~min}$; (4) after $\mathrm{T}=10 \mathrm{~min}$, add $100 \mu \mathrm{l}$ of chromogenic substrate solution (prewarmed at $37^{\circ} \mathrm{C}$ ) to each well; once the substrate solution is added to each well, briefly remove from heating block and gently tap several times; incubate at $37^{\circ} \mathrm{C}$ for $6 \mathrm{~min}$; (5) at $\mathrm{T}=16 \mathrm{~min}$, add $100 \mu \mathrm{l}$ of stop reagent (25\% acetic acid); (6) measure the absorbance at $405-410$ nм on a plate reader.

\section{Cytokine measurement of proinflammatory IL-6 and IL-1 $\beta$}

ELISA was used for quantification of cytokines in blood serum and brain homogenates. Blood was taken from the mouse and incubated for $20 \mathrm{~min}$ at room temperature (RT). After a 15 -min centrifugation at $2000 \times g$ at RT, the blood plasma was collected. Brains were isolated from the same animals.

The brains were homogenized in STKM buffer (250 mM sucrose, 50 mM Tris- $\mathrm{HCl}, 25 \mathrm{~mm} \mathrm{KCl}$, and $5 \mathrm{~mm} \mathrm{MgCl}_{2}$ ) using the GentleMACS (Miltenyi Biotec) program Protein_01. After centrifugation at $4000 \times g$ for $5 \mathrm{~min}$ at $4^{\circ} \mathrm{C}$ the supernatant was centrifuged again for $10 \mathrm{~min}$ at $13,000 \times g$ at $4^{\circ} \mathrm{C}$. Blood serum (1:5) and brain homogenates (1:2; diluted in $1 \%$ BSA solution) were analyzed using the mouse IL- 6 and mouse IL-1 $\beta$ ELISA kit (R\&D Systems).

\section{In vivo NLRP3 inhibition}

C57Bl/6J WT mice were immune stimulated via intraperitoneal injections of $0.2 \mu \mathrm{g} / \mathrm{g}$ LPS of $S$. typhimurium on two consecutive days. Three months after immune stimulation the NLRP3 inhibitor MCC950 (10 $\mu \mathrm{g} / \mathrm{g}$ bodyweight; Avistron Chemistry) was injected intraperitoneally 48 and $24 \mathrm{~h}$ before mice were killed. Each three control $(0.9 \% \mathrm{NaCl})$ and immune-stimulated mice was used for assessment of neuronal structure, function, and microglia as well as astrocyte phenotypes.

\section{DiOlistics and morphologic analysis}

Hippocampal neurons from C57Bl/6J WT, APP/PS1, and NLRP3 KO mice were labeled, three months after immune stimulation using the 
DiOlistic technique on fixed brain slices. Briefly, mice were killed by $\mathrm{CO}_{2}$ asphyxiation and transcardially perfused with $30 \mathrm{ml}$ of $4 \%$ paraformaldehyde and $4 \%$ sucrose in $0.1 \mathrm{~m}$ phosphate buffer. The brains were quickly dissected and incubated in the same fixative for another $30 \mathrm{~min}$ at $4^{\circ} \mathrm{C}$ and cut in $400-\mu \mathrm{m}$-thick coronal sections with a vibratome (Leica VT 1000 S). The lipophilic dye DiI (Invitrogen)-coupled tungsten particles $(1.7 \mu \mathrm{m}$ in diameter; Bio-Rad) were delivered to the fixed brain slices using a hand-held gene gun (Bio-Rad; Helios Gene Gun System) with a pressure of $120 \mathrm{psi}$. A membrane filter ( $3 \mu \mathrm{m}$; Millipore) was used to prevent clusters of large particles from landing on the tissue. Brain slices were incubated in PBS for $24 \mathrm{~h}$ at RT and subsequently stained with 4',6-diamidino-2-phenylindole (DAPI; AppliChem). The neurons were imaged with an Axioplan 2 imaging microscope (Zeiss) equipped with an ApoTome module (Zeiss). Neurons were imaged with a $20 \times$ objective (0.8 N.A., Zeiss) with a z-sectioning of $1 \mu \mathrm{m}$ for assessment of dendritic morphology. Afterwards, mid-apical and basal dendrites were imaged with a BX61WI FluoView 1000 (FV1000) Olympus confocal microscope. Image stacks with z-step size of $0.5 \mu \mathrm{m}$ were acquired using a $40 \times$ oil objective (1.3 NA). Morphologic reconstruction and Sholl analysis of the neurons and their processes were executed with the Neurolucida software (MicroBrightField) and the Neuroexplorer software (MicroBrightField). The density of dendritic spines was determined by counting the number of spines on a defined stretch of dendrite in the ImageJ program (National Institutes of Health). The data obtained were compared between the immune stimulation and respective control conditions using a two-tailed Student's $t$ test. Asterisks indicate the significance levels as follows: ${ }^{*} p<0.05,{ }^{* *} p<0.01$.

\section{Immunohistochemical analysis}

To quantify the inflammatory response in the hippocampus after peripheral immune stimulation, mice were deeply anaesthetized with $\mathrm{CO}_{2}$ and quickly killed via decapitation. Hippocampi were dissected and fixed in $4 \%$ phosphate-buffered paraformaldehyde for $24 \mathrm{~h}$. The fixed tissue was dehydrated in $30 \%$ sucrose in $0.1 \mathrm{~m}$ phosphate buffer. Hippocampi were frozen in Tissue-Tek O.C.T. compound (A. Hartenstein Laborversand) on a freezing microtome and cut into $30-\mu \mathrm{m}$ transversal slices. After blocking with $1 \%$ bovine serum albumin, $0.2 \%$ Triton X-100, and $10 \%$ goat serum in PBS, slices were incubated with the primary antibody mouse anti-GFAP (glial fibrillary acidic protein, 1:1000, Sigma-Aldrich) and rabbit anti-ionizing calcium-binding adapter molecule 1 (IBA-1; $1: 1000$, Synaptic Systems) diluted in $0.2 \%$ Triton X-100 and $10 \%$ goat serum at $4^{\circ} \mathrm{C}$ overnight. The secondary antibodies goat anti-rabbit IgG Cy2 and goat anti-mouse IgG Cy3 (each 1:500, Jackson ImmunoResearch) were diluted in PBS. Nuclei were stained with DAPI solution. GFAP and IBA-1-positive cells were counted in a defined region of interest (ROI) in the stratum radiatum of the CA1 area in overlay with the DAPI channel on image stacks taken with an Axioplan 2 imaging microscope (Zeiss) equipped with an ApoTome module (Zeiss) and $20 \times$ objective (0.8 N.A., Zeiss) with a $\mathrm{z}$-sectioning of $1 \mu \mathrm{m}$. The primary processes emerging from the microglia soma were counted of three microglia per stack on at least three different stacks per mouse using the ImageJ software (National Institutes of Health). Data were calculated as mean and SEM and compared with respective controls via two-tailed Student's $t$ test. Asterisks indicate the significance levels as follows: ${ }^{\star} p<0.05$, ${ }^{* *} p<0.01,{ }^{* *} p<0.001$.

\section{Electrophysiology}

Acute hippocampal slices were prepared from 19-month-old C57Bl/6J WT, APP/PS1, and NLRP3 KO mice three months after peripheral immune stimulation or control treatment. Briefly, mice were deeply anaesthetized by $\mathrm{CO}_{2}$ asphyxiation and quickly killed by decapitation. The brain was removed and rapidly put into $4^{\circ} \mathrm{C}$ cold carbogenated $\left(95 \% \mathrm{O}_{2}, 5 \% \mathrm{CO}_{2}\right.$ ) artificial CSF (ACSF; $124 \mathrm{~mm} \mathrm{NaCl}, 4.9 \mathrm{~mm} \mathrm{KCl}, 1.2$ mм $\mathrm{KH}_{2} \mathrm{PO}_{4}, 2.0 \mathrm{~mm} \mathrm{MgSO}$, $2.0 \mathrm{~mm} \mathrm{CaCl}$, $24.6 \mathrm{~mm} \mathrm{NaHCO}$, and 10 $\mathrm{mm} \mathrm{D}$-glucose). Hippocampi were cut into $400-\mu \mathrm{m}$-thick transversal slices with help of a manual tissue chopper. The slices were incubated on a nylon net in an interface chamber (Scientific system design) and kept under carbonated ACSF bathing at $0.7 \mathrm{ml} / \mathrm{min}$ and $32^{\circ} \mathrm{C}$. After an incubation time of $2 \mathrm{~h}$, one stainless-steel recording electrode ( $5 \mathrm{M} \Omega$; A-M
Systems) and one monopolar lacquer-coated, stainless-steel stimulating electrode ( $5 \mathrm{M} \Omega$; A-M Systems) were placed in the stratum radiatum of the CA1 region to stimulate the Schaffer collateral pathway. First, the input-output relationship was characterized. Therefore, field EPSPs (fEPSPs) were recorded between 100 - and $600-\mu \mathrm{A}$ stimulus intensity in intervals of $100 \mu \mathrm{A}$. Presynaptic properties were analyzed via paired pulse facilitation (PPF). Two stimuli were given at an increasing interstimulus interval (ISI) from 10, 20, 40, 60, 80, and $100 \mathrm{~ms}$, and PPF ratio of the second over the first fEPSP slope was measured at $40 \%$ of the maximum fEPSP slope. LTP was induced after $20 \mathrm{~min}$ of baseline recordings at $40 \%$ of the maximal fEPSP slope via theta burst stimulation (TBS). The TBS contained 10 trains of four pulses at $100 \mathrm{~Hz}$ with an interburst interval of $200 \mathrm{~ms}$, applied three times in 10-s intervals. After induction, LTP was recorded for $60 \mathrm{~min}$ and analyzed as mean \pm SEM in \% fEPSP slope of baseline. Data of each treatment were compared with controls via two-tailed Student's $t$ test. Asterisks indicate the significance levels as follows: ${ }^{*} p<0.05,{ }^{* *} p<0.01,{ }^{* *} p<0.001$.

\section{Morris water maze}

For the analysis of spatial learning in rodents the water maze of Richard Morris was used (Morris, 1984). The water maze pool with $160 \mathrm{~cm}$ in diameter was filled with water at $21 \pm 1^{\circ} \mathrm{C}$ colored opaque with non-toxic white paint (Titanoxid, Euro OTC Pharma), and a white platform was hidden $1 \mathrm{~cm}$ under the water surface in the northwest quadrant of the maze. External cues were provided at an appropriate distance to the pool. To assess swimming and visual abilities of the animals and for the adjustment to the experimental conditions, a pretraining of $3 \mathrm{~d}$ with a cued and visible platform was performed with two trials per day. Mice were either placed on the platform or allowed to stay there for $10 \mathrm{~s}$ at the end of each trial. The platform position was changed for every trial in a random fashion. Mice were trained for eight consecutive days with four trials with a 5-min interval. Each trial lasted maximum $60 \mathrm{~s}$, and the mice were placed or allowed to stay on the platform for another $15 \mathrm{~s}$. The mice were released into the pool from four different positions (Northeast, East, South, Southeast) per day. The mice were tracked with a camera in the ceiling and for each learning day the escape latency, the distance and the used search strategies were analyzed. Search strategies were divided into hippocampus-dependent and hippocampus-independent strategies according to the time spent at the edge of the pool, the annulus zone, or the goal corridor as described by Garthe et al. (2009). Hippocampus-independent strategies were random search ( $>60 \%$ surface coverage), scanning ( $<60 \%$ and $>10 \%$ surface coverage), and chaining ( $>80 \%$ in the annulus zone). Hippocampus-dependent strategies contained directed search $\left(>80 \%\right.$ time in $40^{\circ}$ goal corridor), focal search $(<0.3 \times$ radius to mean distance to the platform, $<0.35 \times$ radius to mean distance to swim path centroid), and direct swimming ( $>90 \%$ in $10^{\circ}$ goal corridor).

To access memory formation, the mice were tested in a memory reference test without the hidden platform on day 3 and day 9 of the training. Here, the time spent in the target quadrant was quantified.

\section{Experimental design and statistical analysis}

For the experimental design in our study, a priori sample size calculations were done using $G^{*}$ power 3.1. All experiments performed and for analysis procedure were done blind to the used mice or pharmacological agent used and code for groups was only revealed to the experimenter, when analysis was ended.

For the comparison of escape latencies in the Morris water maze experiments, a one-way ANOVA was used. The search strategy analysis was conducted with using two-way repeated ANOVA followed by a non-parametric Mann-Whitney $U$ test. Values of $p<0.05$ were considered statistically significant $\left({ }^{*} p<0.05,{ }^{* *} p<0.01,{ }^{* *} p<0.001\right)$. All data are shown as mean \pm SEM.

For the LTP experiments, the data of each treatment were compared with controls via two-tailed Student's $t$ test.

For all experiments, asterisks indicate the significance levels as follows: ${ }^{\star} p<0.05,{ }^{* *} p<0.01,{ }^{* *} p<0.001$. 


\section{Peripheral immune stimulation with LPS in WT mice}
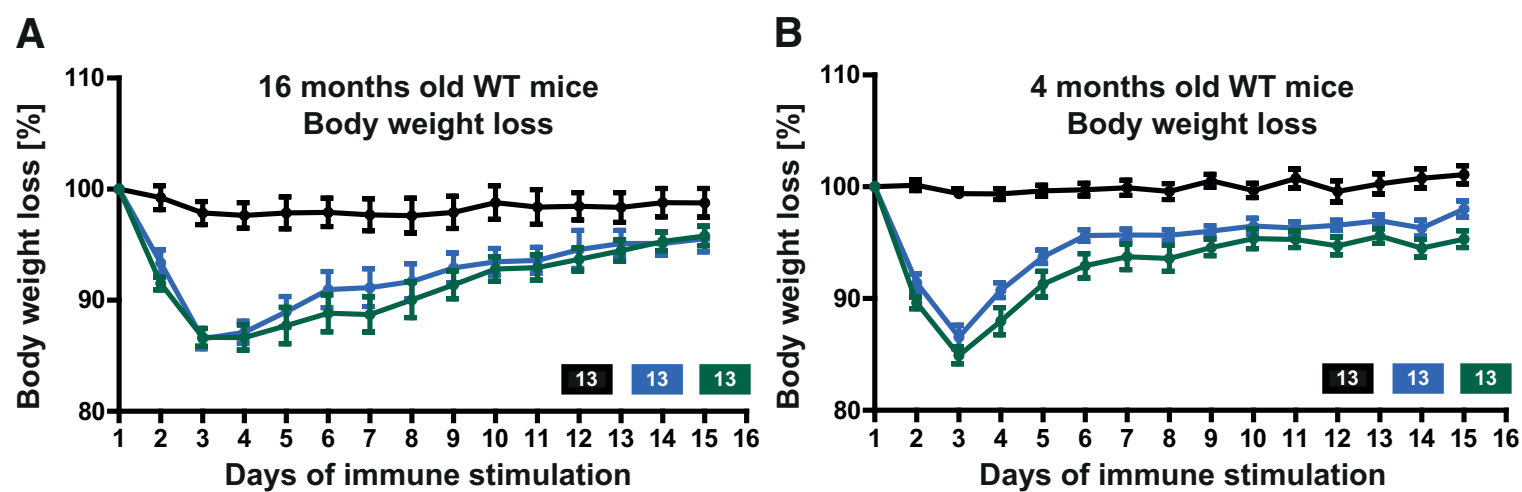

WT CTRL

LPS E.coli

LPS S.typhimurium
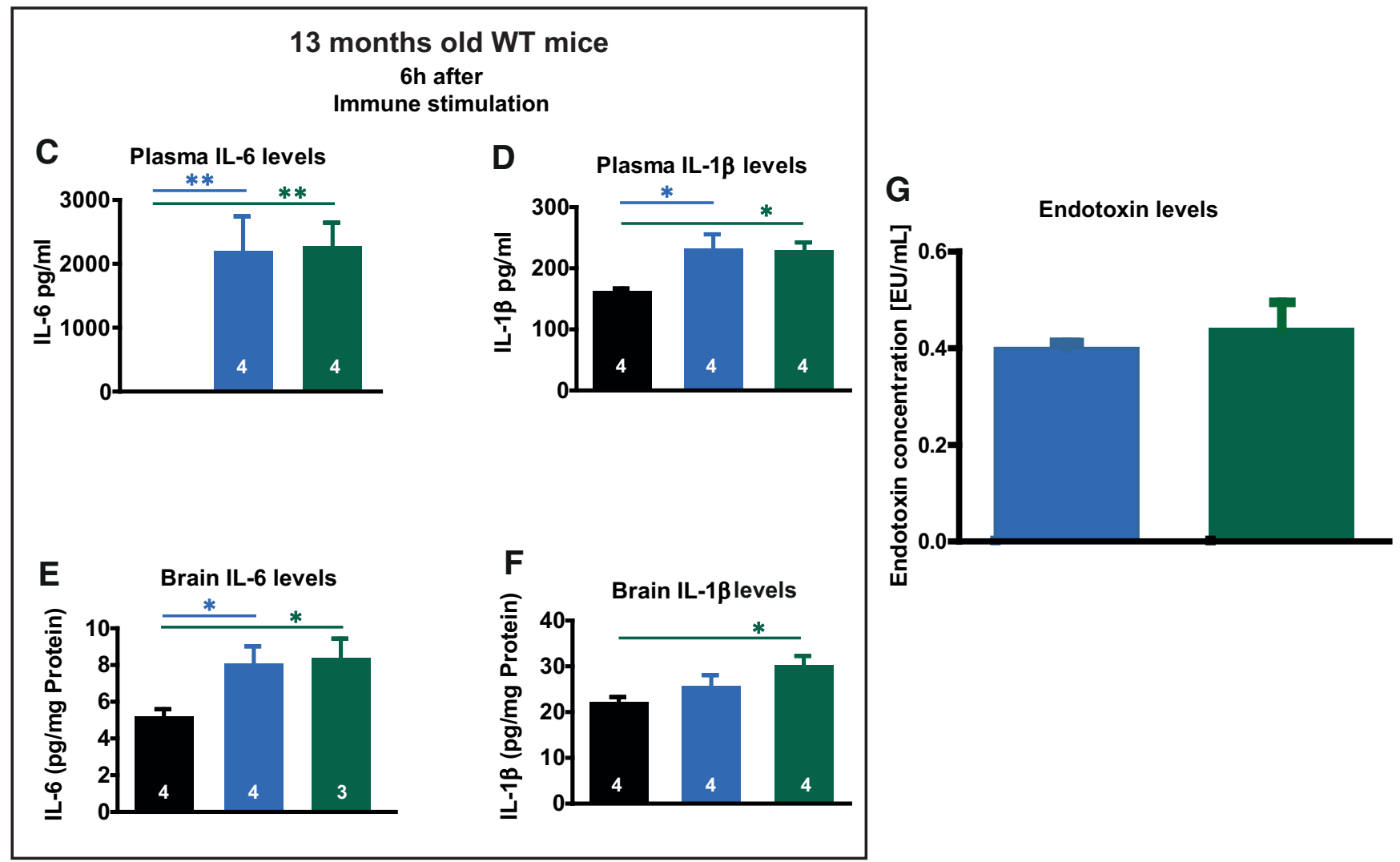

Figure 1. Immune response and body weight loss after peripheral immune stimulation with LPS. $\boldsymbol{A}, \boldsymbol{B}$, Bodyweight loss of aged $(\boldsymbol{A})$ and adult mice $(\boldsymbol{B})$ during $2 \mathrm{~d}$ of peripheral immune stimulation with $0.4 \mu \mathrm{g} / \mathrm{g}$ body weight LPS of $E$. coli (blue) or S. typhimurium (green) and the subsequent $13 \mathrm{~d}$ of recovery. $C, D$, Cytokine levels in blood plasma for IL-6 (C) and IL-1 $\beta$ (D) in 13-month-old mice $6 \mathrm{~h}$ after intraperitoneal injection of $0.2 \mu \mathrm{g} / \mathrm{g}$ body weight LPS from $E$. coli and S. typhimurium. $\boldsymbol{E}, \boldsymbol{F}$, Cytokine levels in brain tissue for IL-6(E) and IL-1 $\beta(\boldsymbol{F})$ in 13 -monthold mice $6 \mathrm{~h}$ after immune stimulation. $\boldsymbol{G}$, Endotoxin concentration in EU/ml comparing LPS of $E$. coli and LPS of S. typhimurium. $\boldsymbol{A}, \boldsymbol{B}, N=13$ mice per group; $\boldsymbol{C}-\boldsymbol{H}, \boldsymbol{N}=3-4$ mice per group; all data are presented as mean \pm SEM; ${ }^{*} p<0.05,{ }^{* *} p<0.01$.

\section{Results}

Response to peripheral immune stimulation with two different kinds of LPS

Bacterial LPS is widely used to induce a strong but transient peripheral immune response including increase of proinflammatory cytokines. Furthermore, it was shown that although the peripheral cytokines or the LPS itself do not diffuse across the blood brain barrier (Wendeln et al., 2018), the systemic inflammation leads to neuroinflammation mediated by glia cells (Qin et al., 2007; Biesmans et al., 2013).

We used two different types of LPS, from E. coli or S. enterica serotype typhimurium with similar endotoxic capacity (Fig. $1 G$ ), to induce a rapid sickness behavior and body weight loss with its peak $24 \mathrm{~h}$ after immune stimulation and subsequent recovery until day 15 in adult (four months) as well as aged (1316 months) C57Bl/6J WT mice (Fig. $1 A, B$ ). As fast as $6 \mathrm{~h}$ after LPS injection, the plasma levels of IL- 6 and IL- $1 \beta$ were highly and significantly increased within the same degree with both types of LPS (WT 13 months 6 h IL- 6 one-way ANOVA $F_{(2,9)}=$ 12.14; $p=0.003$ : LPS E. coli Fisher's LSD $p=0.0023$; LPS S. typhimurium Fisher's LSD $p=0.0019 ;$ IL- $\beta$ one-way ANOVA $F_{(2,9)}=6.148 ; p=0.0207$ : LPS E. coli Fisher's LSD $p=0.0132$; LPS $S$. typhimurium Fisher's LSD $p=0.0151$; Fig. $1 C, D)$. In the brain tissue of the same mice, the concentration of IL- 6 went up again 
in similar fashion by LPS of E. coli and S. typhimurium. The IL$1 \beta$ concentration was increased with both types of LPS as well, but the $S$. typhimurium LPS caused a stronger phenotype (WT 13 months 6 h IL- 6 one-way ANOVA $F_{(2,8)}=5.013 ; p=0.0388$ : LPS E. coli Fisher's LSD $p=0.0277$; LPS S. typhimurium Fisher's LSD $p=0.0258 ;$ IL- $\beta$ one-way ANOVA $F_{(2,9)}=4.272$; $p=0.0496$ : LPS E. coli Fisher's LSD $p=0.2277$; LPS S. typhimurium Fisher's LSD $p=0.0171$; Fig. $1 E, F)$.

\section{Prolonged microglia activation in the brain of aged immune- stimulated mice}

To examine the possibly long-lasting activation of brain resident cells involved in innate immunity after peripheral immune stimulation with LPS, we stained microglia and astrocytes in the hippocampi of adult and aged WT mice $24 \mathrm{~h}$ and three months after LPS injections. At $24 \mathrm{~h}$, the number of IBA1-positive microglia/ macrophages was significantly elevated in the stratum radiatum of the CA1 region of the hippocampus only on stimulation with LPS from $S$. typhimurium and only in aged mice (WT 16 months 24 h one-way ANOVA $F_{(2,26)}=3.373 ; p=0.0498$ : LPS S. typhimurium Fisher's LSD $p=0.0393$; WT four months 24 h one-way ANOVA $F_{(2,27)}=1.484 ; p=0.2447$; Fig. $\left.2 A, D, J\right)$. The activation status of the microglia/macrophages, however, as quantified by the number of primary branches, was altered in the hippocampi of adult and aged mice by both types of LPS (WT 16 months 24 h one-way ANOVA $F_{(2,26)}=16.21 ; p<0.0001$ : LPS E. coli Fisher's LSD $p=0.0001$; LPS $S$. typhimurium Fisher's LSD $p<0.0001$; WT fourmonths $24 \mathrm{~h}$ one-way ANOVA $F_{(2,26)}=$ 10.89; $p=0.0004$ : LPS E. coli Fisher's LSD $p=0.0091$; LPS $S$. typhimurium Fisher's LSD $p<0.0001$; Fig. $2 F, L)$. Counting of GFAPpositive astrocytes revealed an increase of cell numbers in adult and aged mice and again independent of the LPS component used (WT 16 months $24 \mathrm{~h}$ : control mice (CTRL) $1.00 \pm 0.08$; LPS E. coli $1.41 \pm 0.10, p=0.0063$; LPS $S$. typhimurium $1.31 \pm 0.06, p=0.0081, t=4.141 \mathrm{df}=31$; WT four months $24 \mathrm{~h}$ : CTRL $1.00 \pm 0.05$; LPS E. coli $1.29 \pm 0.06, p=0.0012, t=4.39$ $\mathrm{df}=34$; LPS $S$. typhimurium $1.27 \pm 0.07, p=0.0042, t=3.245$ $\mathrm{df}=27$; Fig. $2 H, N)$. Three months after peripheral immune stimulation, the astrocyte numbers were not distinguishable from control animals any longer in both adult and aged mice (Fig. 2I, $O)$. A prolonged activation of microglia cells was observed in aged mice three months after $S$. typhimurium LPS application. Here, the number and activation was still significantly increased (WT 16 months-3months after immune stimulation cell number one-way ANOVA $F_{(2,38)}=5.908 ; p=0.0058$ : LPS $S$. typhimurium Fisher's LSD $p=0.0015$; primary processes one-way ANOVA $F_{(2,38)}=20.26 ; p<0.0001$ : LPS E. coli Fisher's LSD $p<0.0001$; LPS $S$. typhimurium Fisher's LSD $p<0.0001$; Fig. 2E,G). In adult mice, however, neither the number of microglia nor the activation of microglia itself was altered three months after immune stimulation with LPS when compared with non-stimulated age-matched controls (WT 4 months-3months after immune stimulation cell number oneway ANOVA $F_{(2,22)}=1.015 ; p=0.3787$; primary processes oneway ANOVA $F_{(2,22)}=0.6714 ; p=0.5212$; Fig. $\left.2 K, M\right)$. To explain these age-dependent differences, we compared the microglia in the adult and aged WT control mice (CTRL). Interestingly, while the numbers of microglia were independent of age, 19-monthold mice showed a higher activation of microglia in comparison to their younger, seven-month-old counterparts (age-dependent changes cell number: adult WT; aged WT unpaired $t$ test $t=1.469 \mathrm{df}=16 ; p=0.1612$; primary processes: adult WT; aged WT unpaired $t$ test $t=4.708 \mathrm{df}=16 ; p=0.0002$; Fig. $2 B, C$ ).

\section{LPS from E. coli and S. typhimurium negatively affect neuronal morphology in aged mice}

To determine the long-term influences of a systemic immune stimulation on the architecture of excitatory neurons in the hippocampus, one of the most vulnerable regions in the brain, dendritic complexity and spine density of CA1 pyramidal neurons and dentate gyrus (DG) granule cells of adult (seven months) and aged (19 months) C57Bl/6J WT mice were analyzed three months after intraperitoneal injection of LPS from E. coli or $S$. typhimurium. In aged animals, E. coli LPS caused a reduction of dendritic complexity of the CA1 proximal apical dendritic tree (Fig. 3A) as well as the spine density of mid-apical dendrites (WT 19 months CA1 apical one-way ANOVA $F_{(2,44)}=3.797$; $p=0.0301$ : LPS E. coli Fisher's LSD $p=0.0333$; LPS S. typhimurium Fisher's LSD $p=0.0151$; Fig. $3 D$ ). The granule cells of the DG manifested an even higher dendritic complexity in response to E. coli LPS (Fig. 3B). The treatment with LPS from S. typhimurium resulted in significantly lower complexity of proximal basal dendrites and spine densities of apical and basal CA1 dendrites (WT 19 months CA1 basal complexity two-way ANOVA $F_{(2,26)}=$ 2.281; $p=0.1222$ : LPS $S$. typhimurium Turkey's distance $30 \mu \mathrm{m}$ $p=0.0353$; WT 19 months CA1 apical two-way ANOVA $F_{(2,26)}=$ 1.279; $p=0.2954$ : LPS E. coli Turkey's distance 60-110 $\mu \mathrm{m}$ $p<0.022$; LPS S. typhimurium Turkey's distance 60-90 $\mu \mathrm{m}$ $p<0.03$; WT 19 months CA1 basal spines one-way ANOVA $F_{(2,39)}=2.877 ; p=0.0683$ : LPS $S$. typhimurium Fisher's LSD $p=0.0228$; WT 19 months CA1 apical spines one-way ANOVA $F_{(2,44)}=3.797 ; p=0.0301$ : LPS $E$. coli Fisher's LSD $p=0.0333$; LPS $S$. typhimurium Fisher's LSD $p=0.0151$; Fig. $3 A, C, D)$, while the dendritic complexity of the granule cells was on control level (WT 19 months DG two-way ANOVA $F_{(2,42)}=2.179 ; p=0.1258$ : LPS E. coli Turkey's distance 150-170 $\mu \mathrm{m} p<0.042$; Fig. $3 B$ ). Here, the spine density was only slightly reduced (WT 19 months DG one-way ANOVA $F_{(2,45)}=1.281 ; p=0.2878$; Fig. $3 E$ ). In adult mice, neither the dendritic architecture nor the spine density of CA1 pyramidal neurons was altered on immune stimulation with LPS from either E. coli or S. typhimurium (WT seven months CA1 basal two-way ANOVA $F_{(2,26)}=0.4034 ; p=0.6722 ;$ CA1 apical two-way ANOVA $F_{(2,21)}=1.020 ; p=0.3778$; WT seven months CA1 basal spines one-way ANOVA $F_{(2,43)}=0.1603 ; p=0.8524$; WT seven months CA1 apical spines one-way ANOVA $F_{(2,43)}=0.4223$; $p=0.6583$; Fig. $3 F, H, I)$. In contrast, the granule cells of the DG display an even higher dendritic complexity on LPS S. typhimurium treatment in the distal dendritic tree (Fig. $3 G$ WT seven months DG two-way ANOVA $F_{(2,42)}=1.988$; $p=0.1496$ : LPS $S$. typhimurium Turkey's distance 160 and $180 \mu \mathrm{m}$ $p<0.02$ ). The spine density is again not altered (WT seven months DG one-way ANOVA $F_{(2,37)}=0.8299 ; p=0.4440$; Fig. $\left.3 J\right)$.

\section{LPS of S. typhimurium leads to impairment of synaptic plasticity in aged mice}

Short-term as well as long-term plasticity were investigated at the CA3-CA1 Schaffer collaterals in hippocampal acute slices of 16- to 19-month-old C57Bl/6J WT mice three months after systemic immune stimulation. While E. coli LPS did not affect LTP, the induction as well as the maintenance of LTP was significantly impaired at all time points of the recording after S. typhimurium LPS treatment (WT 19 months mean LTP two-way ANOVA $F_{(2,41)}=$ v5.909; $p=0.0056$; last $10 \mathrm{~min}$ LTP one-way ANOVA $F_{(2,41)}=3.334 ; p=0.0455:$ LPS S. typhimurium Fisher's LSD $p=0.0704$; $t$ test $t=2.456 \mathrm{df}=31 ; p=0.0198$; Fig. $4 A$ ). To examine whether the defect in LTP was caused by impaired basal 


\section{Morphological changes in glia cells after immune stimulation}
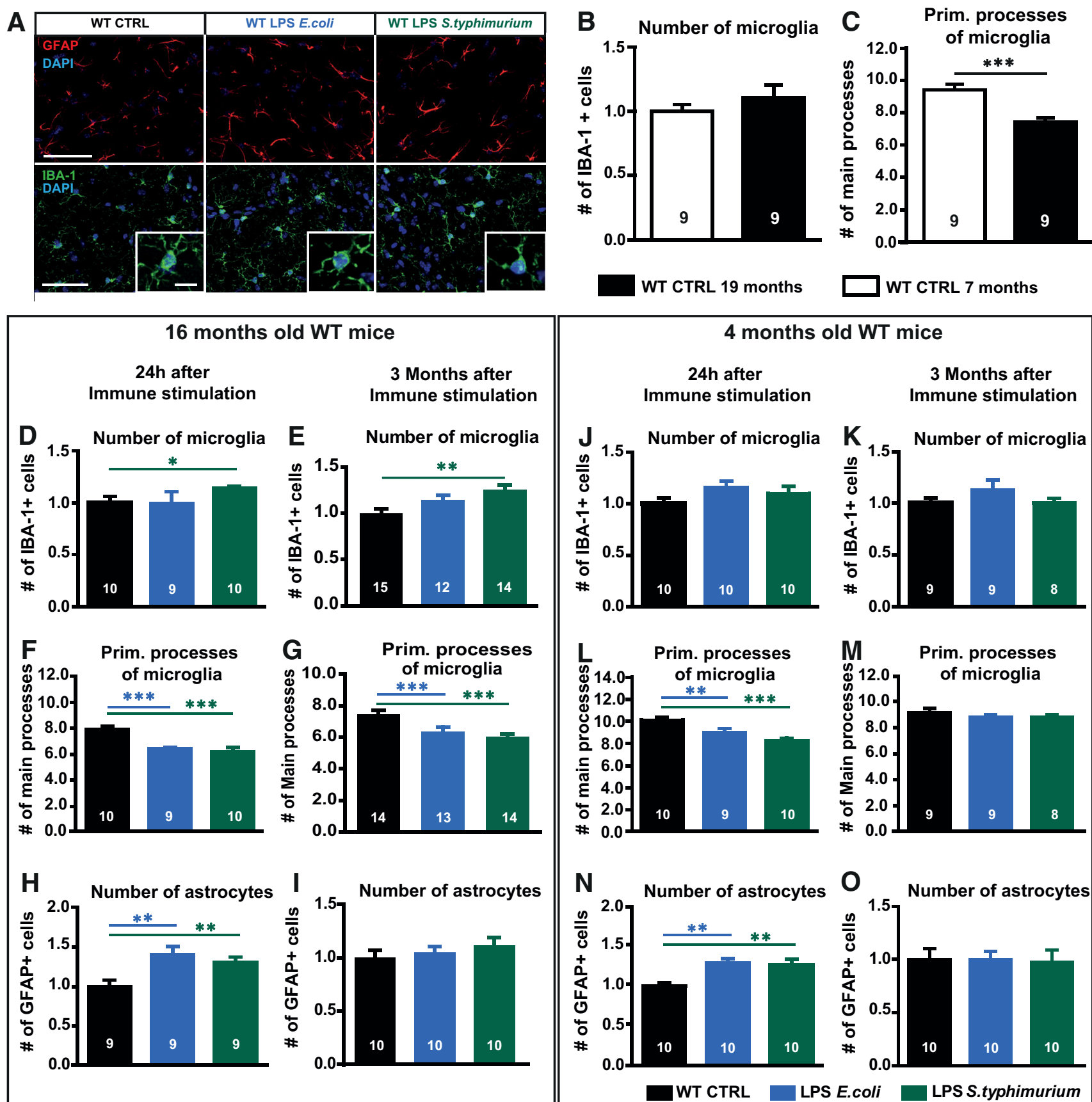

Figure 2. Astrocyte and microglia phenotype of adult and aged WT mice after immune stimulation with $0.4 \mu \mathrm{g} / \mathrm{g}$ bodyweight LPS of $E$. coli or $S$. typhimurium. $\boldsymbol{A}$, Immunohistochemical staining of GFAP (red) and IBA-1 (green) in the CA1 stratum radiatum in overlay with DAPI (blue), scale bar large frame $100 \mu \mathrm{m}$, smaller detail $10 \mu \mathrm{m}$. $\boldsymbol{B}$, Relative number of IBA-1-positive cells of control-injected adult (seven months, white bar) and aged mice (16-19 months, black bar), normalized to adult controls. C, Quantification of primary branches of IBA-1-positive cells of control-injected adult and aged mice (seven vs 16-19 months). $\boldsymbol{D}, \boldsymbol{E}, \boldsymbol{J}, \boldsymbol{K}$, Relative number of IBA-1-positive cells of immune-stimulated mice (LPS E. coli blue, LPS S. typhimurium green) compared with aged controls (black), $24 \mathrm{~h}(\boldsymbol{D})$, and three months $(\boldsymbol{E})$ after immune stimulation and in adult mice after $24 \mathrm{~h}(\boldsymbol{J})$, three months $(\boldsymbol{K})$, normalized to respective controls. $\boldsymbol{F}, \boldsymbol{G}, \boldsymbol{L}, \boldsymbol{M}$, Number of primary branches of IBA-1-positive cells in control and immune-stimulated aged mice, $24 \mathrm{~h}(\boldsymbol{F})$ and three months $(\boldsymbol{G})$ after immune stimulation and adult mice $24 \mathrm{~h}(\boldsymbol{L})$ and three months $(\boldsymbol{M}) . \boldsymbol{H}, \boldsymbol{I}, \boldsymbol{N}, \mathbf{0}$, Quantification of GFAP-positive cells in aged mice, $24 \mathrm{~h}(\boldsymbol{H})$ and three months $(\boldsymbol{I})$ after immune stimulation, normalized to aged controls and $24 \mathrm{~h}(\boldsymbol{N})$ and three months $(\boldsymbol{O})$ in adult mice, normalized to the respective adult controls; $N=3$ mice per group ( $n=8-10), N=4$ or 5 mice in $\boldsymbol{E}, \boldsymbol{G}, \boldsymbol{I}(n=12-15)$; $n=$ number of samples; all data are presented as mean \pm SEM; ${ }^{*} p<0.05,{ }^{* *} p<0.01,{ }^{* * *} p<0.001$.

synaptic transmission, the input-output properties of the hippocampal CA3-CA1 Schaffer collateral pathway were evaluated. Here, with increasing stimulus intensities also the negative fEPSP slope increased independently of the different treatments. While there is a perfect overlap of input-output (I/O) curves under control and LPS S. typhimurium-treated conditions, surprisingly the slices of $E$. coli LPS-treated animals showed significantly lower response at 

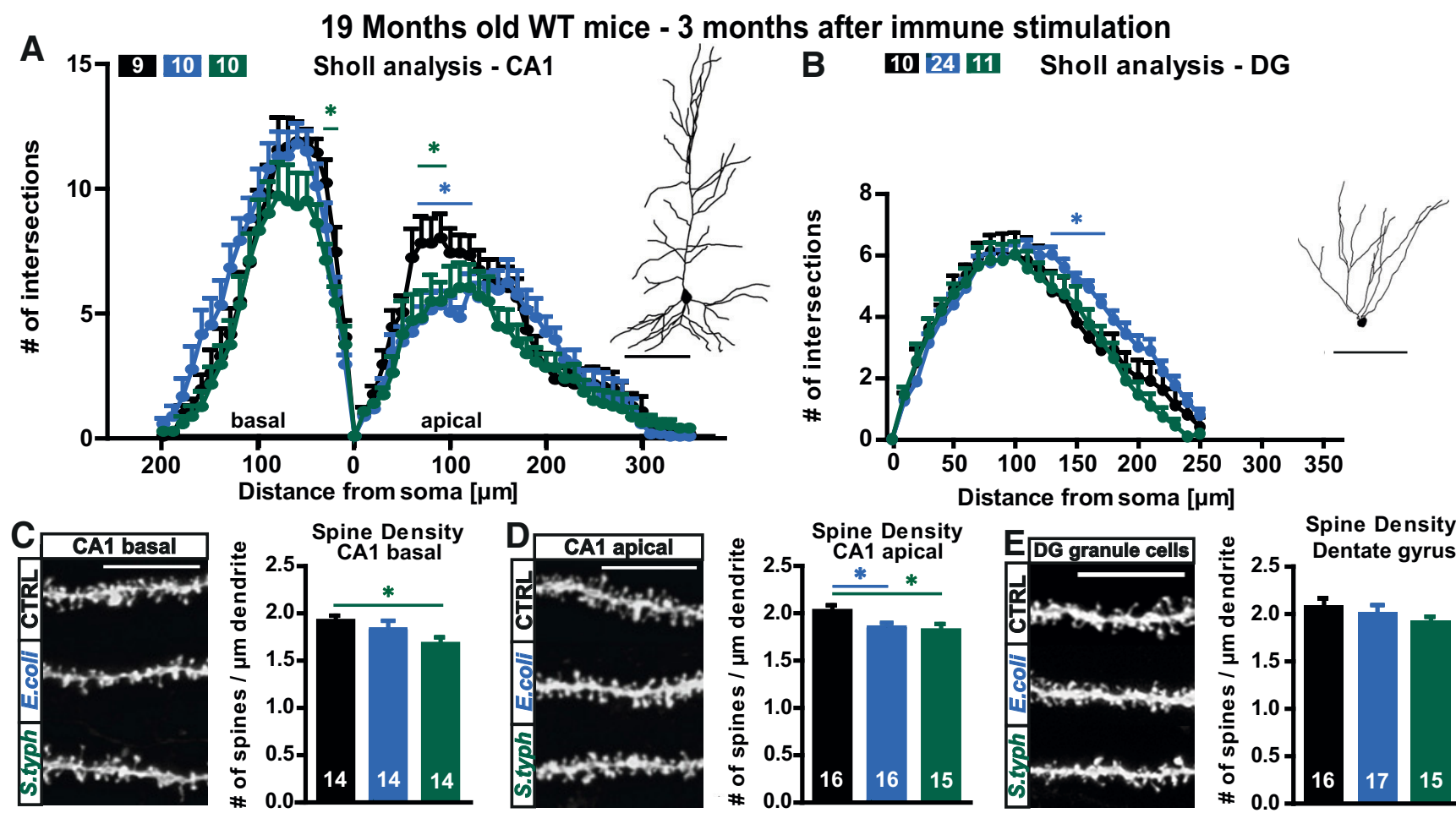

7 Months old WT mice - 3 months after immune stimulation

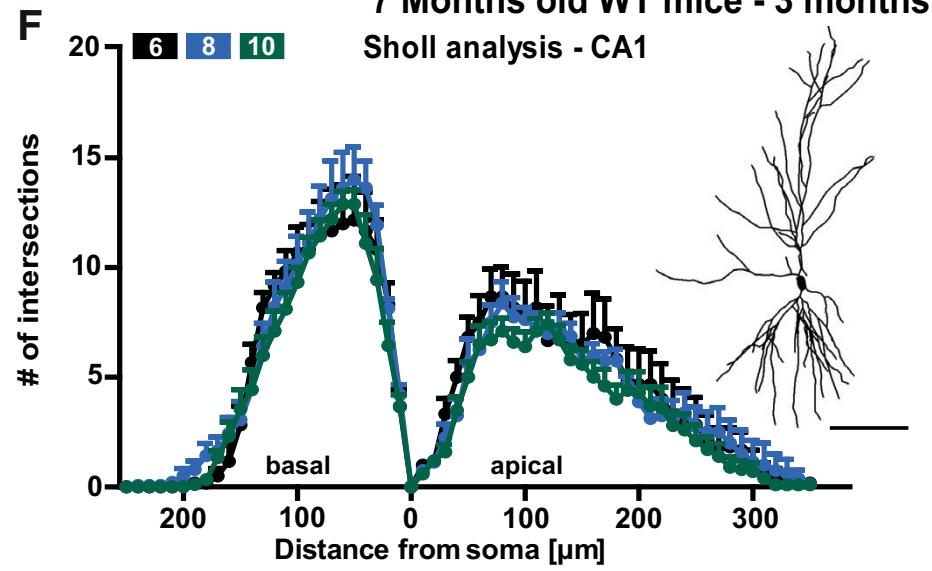

G 151911 Sholl analysis - DG
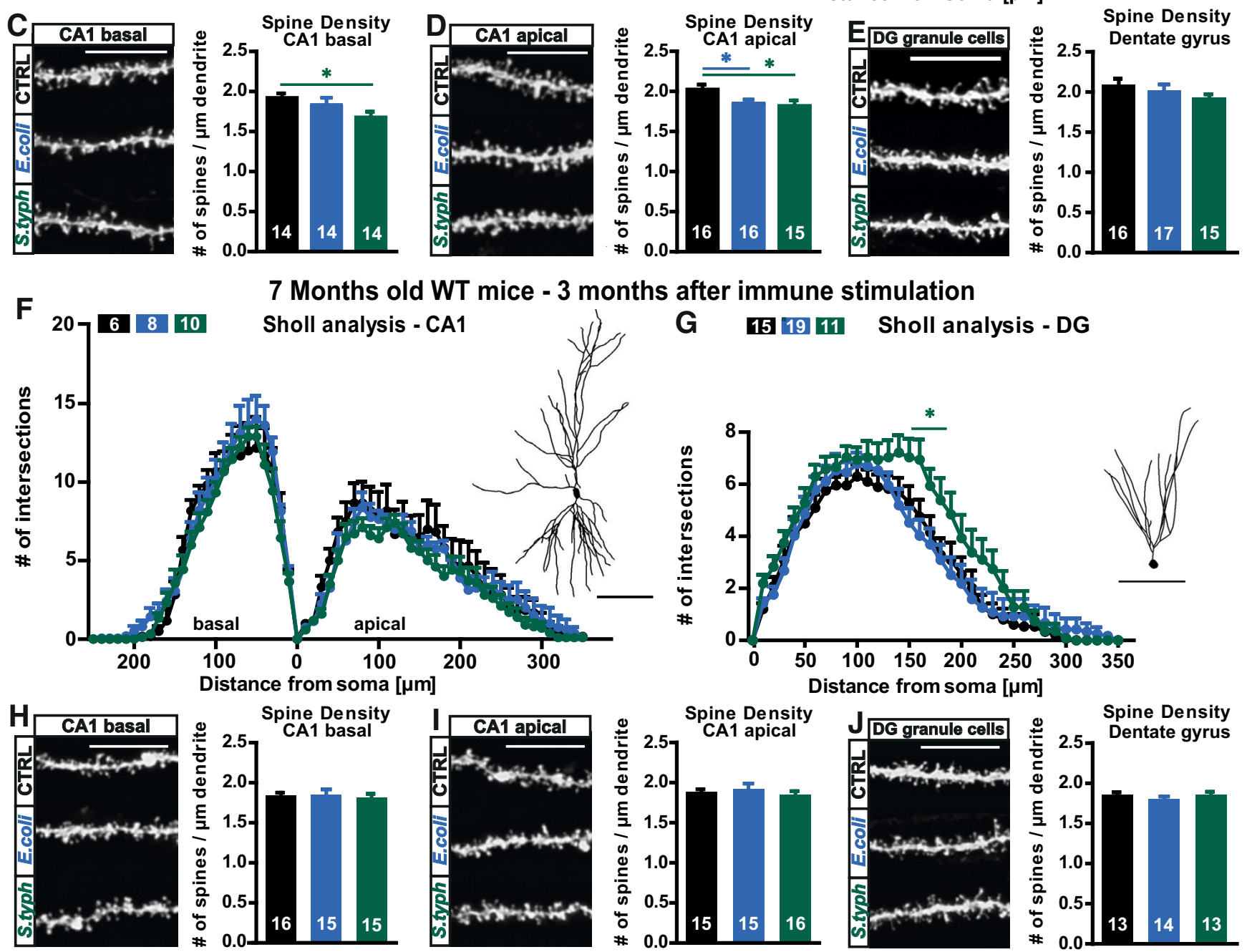

Spine Density

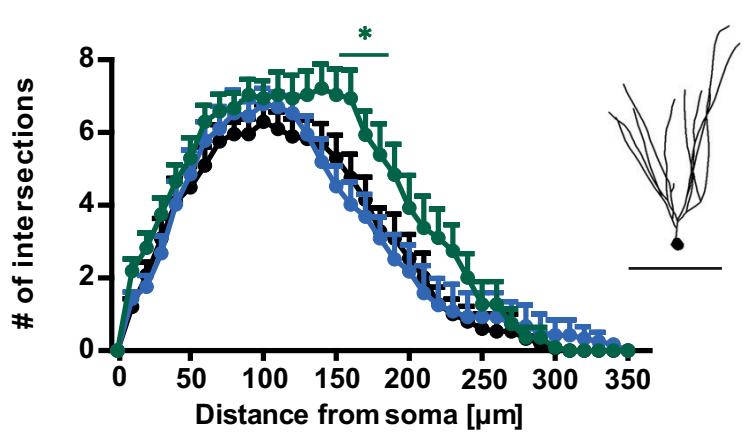

WT CTRL WT LPS E.coli WT LPS S.typhimurium

Figure 3. Neuronal morphology of adult and aged WT mice three months after immune stimulation with $0.4 \mu \mathrm{g} / \mathrm{g}$ body weight LPS of $E$. coli or $S$. typhimurium. A-E, Neuronal architecture of 16 to 19-month-old WT mice, control (black), LPS E. coli (blue), and LPS S. typhimurium (green): tracings and Sholl analysis of (A) CA1 pyramidal neurons and (B) DG granule cells. Confocal microscopic images and spine density analysis from $(\boldsymbol{D})$ apical CA1 dendrites, $(\boldsymbol{C}$ basal CA1 dendrites, and $(\boldsymbol{E})$ granule cells from the $D G$. $\boldsymbol{F}-\boldsymbol{J}$, Neuronal architecture of seven-month-old WT mice, control (black), LPS E. coli (blue), and LPS S. typhimurium (green): dendritic complexity and respective tracing of (F) CA1 hippocampal neurons and (G) DG granule cells. Spine density and confocal images of typical dendrites from ( () apical CA1 dendrites, $(\boldsymbol{H})$ basal CA1 dendrites, and (I) DG granule cells; $N=3(\boldsymbol{A}, n=9-10 ; \boldsymbol{B}, n=10-24 ; \boldsymbol{C}, n=14 ; \boldsymbol{D}, n=15-16 ; \boldsymbol{E}, n=15-17 ; \boldsymbol{F}, n=6-14 ; \boldsymbol{G}, n=11-19 ; \boldsymbol{H}-\boldsymbol{J}$, $n=13-16) ; n=$ number of samples, scale bar of tracings, $100 \mu \mathrm{m}$; scale bar of confocal fluorescent images, $10 \mu \mathrm{m}$; all data are presented as mean \pm SEM, ${ }^{*} p<0.05$.

stimulus intensities of $200-600 \mu \mathrm{A}$ in comparison to controls (I/O two-way ANOVA $F_{(2,65)}=4.793 ; p=0.0114$ : LPS $E$. coli Turkey's 300-600 $\mu \mathrm{A} p<0.006$; Fig. 4B). This might be caused by a decreased apical CA1 complexity (Fig. $3 A$ ), but, however, it had no impact on LTP. To address the presynaptic properties of the pathway, PPF was measured. With both kinds of LPS, a PPF curve at control level was achieved (PPF two-way ANOVA $F_{(2,26)}=0.7372 ; p=0.4882$; Fig. $\left.4 C\right)$. 


\section{9 months old WT mice}
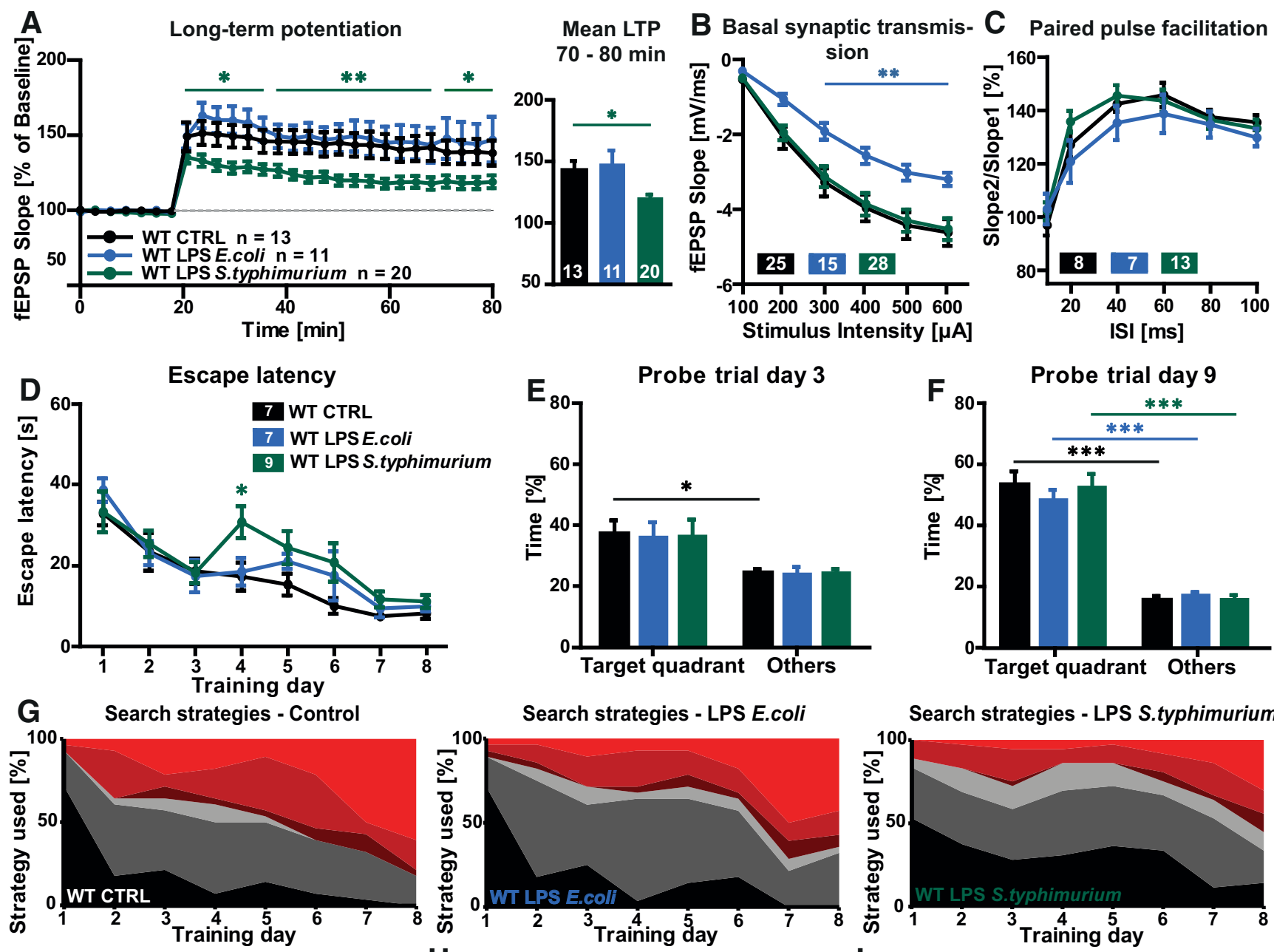

Spatial learning

H Hippocampus-dependent strategies

HC-dependent strategies - first trail only

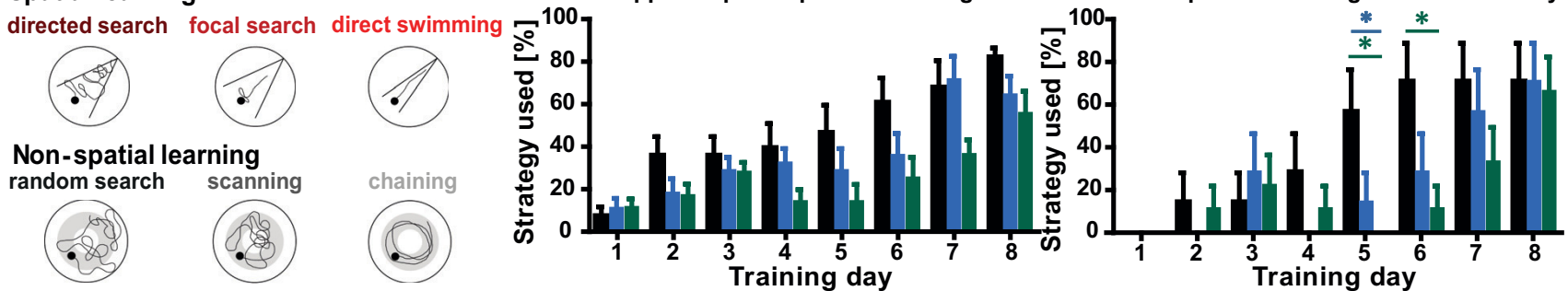

Figure 4. Synaptic plasticity and spatial learning in immune-stimulated aged WT mice. $\boldsymbol{A}-\boldsymbol{C}$, Short-term and long-term synaptic plasticity at the CA3-CA1 pathway in hippocampal acute slices of aged WT mice three months after peripheral immune stimulation, controls (black), LPS E. coli ( $0.4 \mu \mathrm{g} / \mathrm{g}$ bodyweight, blue), and LPS S. typhimurium ( $0.4 \mu \mathrm{g} / \mathrm{g}$ bodyweight, green): (A) LTP induced via TBS after $20 \mathrm{~min}$ of baseline recordings and mean LTP fEPSP slope in \% for 70-80 min of recording. $\boldsymbol{B}$, Input-output curve. C, PPF with increasing ISIs; $N=3-4$ mice per group, $n=7-28 ;{ }^{*} p<0.05,{ }^{* *} p<0.01$. D-I, Nineteen-month-old WT were challenged in the Morris water maze navigation task, controls (black), LPS E. coli (blue), and LPS S. typhimurium (green): (D) escape latency, mean values of four trials per mouse per day. $\boldsymbol{E}, \boldsymbol{F}$, Target quadrant preference in comparison to time spent in other quadrants during memory reference tests (probe trials) on training day $3(\boldsymbol{E})$ and day $9(\boldsymbol{F})$. $\boldsymbol{G}$, Illustration and quantification of the six different search strategies random search, scanning, chaining, directed search, focal search, and direct swimming as mean of four trials per mouse per day. $\boldsymbol{H}$, Quantification of the hippocampus-dependent search strategies for every single training day, mean value of four trials per mouse and day. $\mathbf{I}$, Quantification of the hippocampus-dependent search strategies for every single training day during the first trial; $N=7-9$, all data shown as mean $\pm S E M ;{ }^{*} p<0.05,{ }^{* * *} p<0.001 ; n=$ number of mice.

Taken together, treatment with LPS of S. typhimurium leads to an LTP defect, which cannot be explained with impaired basal synaptic transmission or defects in PPF.

LPS S. typhimurium affects spatial learning of aged WT mice The Morris water maze navigation task used in this study to evaluate the behavioral consequences of a peripheral immune stimulation was designed to test spatial learning in rodents (Morris, 1984). Independently of the treatment, all 19-month-old WT mice managed to learn the position of the hidden platform in the Morris water maze arena as a decrease of the escape latency was documented (WT 19 months escape latency two-way ANOVA $F_{(2,20)}=2.089 ; p=0.1500$ : day 4 LPS S. typhimurium Turkey's $p=0.0138$; Fig. $4 D$ ). Interestingly, although the escape latencies decreased in both controls and LPS S. typhimurium-treated mice about the same degree during the whole training, the immunestimulated mice took a significantly longer time to find the hidden platform on day 4 of the training period. To test memory 
formation during the Morris water maze, memory reference experiments (probe trials) were performed on the third and ninth day of the training period. In absence of the hidden platform, control as well as LPS-treated mice spent more time in the target quadrant with the former platform, when compared with the other quadrants which was statistically significant for the control group (WT 19 months CTRL PT day 3: other quadrants; target quadrant unpaired $t$ test $t=2.681 \mathrm{df}=12 ; p=0.0200$; Fig. $4 E$ ). In the probe trial on day 9 , all mice spent clearly and highly significantly more time in the target quadrant (WT 19 months CTRL PT day 9: other quadrants; target quadrant unpaired $t$ test $t=8.007 \mathrm{df}=12 ; p<0.0001$; LPS E. coli: other; target unpaired $t$ test $t=8.686 \mathrm{df}=12 ; p<0.0001$; LPS $S$. typhimurium: other; target unpaired $t$ test $t=7.792 \mathrm{df}=16$; $p<0.0001$; Fig. $4 F$ ).

To emphasize possible differences in the learning behavior of the immune-stimulated mice, the search strategies during the learning period were analyzed (Fig. $4 G, H$ ). In control mice, the usage of all hippocampus-independent strategies decreased progressively, especially in favor of direct swimming. Also, the LPS E. coli-treated mice showed a steady decrease of random search and scanning and a steady increase of direct swimming, which was less pronounced than in control mice. In the LPS S. typhimurium-treated mice, however, although there was a slight increase of hippocampus-dependent learning from day 1 to day 3 of the training, on day 4 and 5 , again there was no change in the proportion of the six evaluated search strategies in comparison to day 1 (Fig. 4G, right diagram). Only from day 6 to day 8 of learning, random search and scanning were used more seldom. Therefore, LPS S. typhimurium-treated mice showed a less pronounced hippocampus-dependent learning progression. During the training period, mice performed four trials per day. While the second, third, and fourth trial assessed long-term as well as working memory, only the first trial displays long-term memory formation. When the first search trial per mouse was analyzed, the differences in the use of hippocampus-dependent (HC) strategies between control and the S. typhimurium LPS-treated mice were even more obvious (HC: Hippocampus-dependent strategies, WT 19 months day 5 one-way ANOVA $F_{(2,20)}=5.217 ; p=0.0150$ : LPS E. coli Turkey's $p=0.0325$; LPS $S$. typhimurium Turkey's $p=0.0228$; day 6 oneway ANOVA $F_{(2,20)}=3.928 ; p=0.0364$ : LPS E. coli Turkey's $p=0.1786$; LPS S. typhimurium Turkey's $p=0.0307$; Fig. $4 I$ ).

\section{Neuronal morphology and function are altered on stimulation with E. coli and S. typhimurium LPS in APP/PS1 mice}

We further investigate the question how a brain with pathologic hallmarks of $\mathrm{AD}$ reacts to a peripheral immune challenge. In order to investigate whether an immune stimulation of a murine $\mathrm{AD}$ model serves as a second proinflammatory stimulus to the already challenged and proinflammatory primed brain, together leading to an even more severe neurodegenerative phenotype, adult and aged APP/PS1 mice (Borchelt et al., 1997; Jankowsky et al., 2004a) were peripherally immune stimulated as described above for WT mice. In the basal compartment of CA1 neurons of the hippocampus, LPS from E. coli and S. typhimurium both caused a reduction in dendritic complexity (APP/PS1 19 months CA1 basal two-way ANOVA $F_{(2,29)}=1.355 ; p=0.2739$ : LPS $E$. coli Turkey's distance 20-70 $\mathrm{mm} p<0.03$; LPS S. typhimurium Turkey's distance 30-70 $\mu \mathrm{m} p<0.015$ and distance $120-160 \mu \mathrm{m}$ $p<0.05 ;$ APP/PS1 19 months CA1 apical two-way ANOVA $F_{(2,30)}=0.1667 ; p=0.8472$; Fig. $\left.5 A\right)$. Architecture of apical dendrites was not altered. In contrast to the dendritic level, the more severe changes on the synaptic level occurred in the apical dendritic tree. Here, the spine density was highly significantly reduced with both kinds of LPS when compared with control conditions (APP/PS1 19 months CA1 apical one-way ANOVA $F_{(2,33)}=11.36 ; p=0.0002$ : LPS $E$. coli Fisher's LSD $p=0.0010$; LPS S. typhimurium Fisher's LSD $p<0.0001$; Fig. $5 D)$. The basal spine density was significantly reduced only on stimulation with LPS from $E$. coli in aged APP/PS1 mice (APP/PS1 19 months CA1 basal one-way ANOVA $F_{(2,31)}=2.948 ; p=0.0673:$ LPS $E$. coli Fisher's LSD $p=0.0402$; Fig. $5 C$ ). The granule cells of the DG exhibited a reduced number of intersections in the Sholl analysis on treatment with either LPS from E. coli or S. typhimurium (APP/PS1 19 months DG two-way ANOVA $F_{(2,27)}=4.149$; $p=0.0268$ : LPS E. coli Turkey's distance 140-200 $\mu \mathrm{m} p<0.03$; LPS S. typhimurium Turkey's distance 140-160 $\mu \mathrm{m} p<0.028$; Fig. $5 B$ ), and also the spine density of these cells was significantly reduced $\left(\mathrm{APP} / \mathrm{PS} 119\right.$ months $\mathrm{DG}$ one-way $\mathrm{ANOVA} F_{(2,32)}=$ 5.378; $p=0.0097$ : LPS $E$. coli Fischer's LSD $p=0.0707 ; t$ test $t=2.115 \mathrm{df}=18 ; p=0.0486$; LPS $S$. typhimurium Fischer's LSD $p=0.0025$; $t$ test $t=3.251 \mathrm{df}=23$; $p=0.0035$; Fig. $5 E$ ).

The dendritic complexity of the CA1 pyramidal neurons of seven-month-old APP/PS1 mice showed no differences on immune stimulation with E. coli or S. typhimurium LPS (Fig. 5I), and only the basal CA1 spine density was significantly reduced by $S$. typhimurium LPS (APP/PS1 seven months CA1 basal oneway ANOVA $F_{(2,36)}=6.803 ; p=0.0031$ : LPS $S$. typhimurium Fisher's LSD $p=0.0072$; Fig. $5 K)$. The DG showed a reduced spine density with both kinds of LPS (APP/PS1 seven months DG one-way ANOVA $F_{(2,38)}=6.381 ; p=0.0041$ : LPS E. coli Fischer's LSD $p=0.0954 ; t$ test $t=2.190 \mathrm{df}=23 ; p=0.0389$; LPS $S$. typhimurium Fisher's LSD $p=0.0010$; $t$ test $t=3.427 \mathrm{df}=29$; $p=0.0018$; Fig. $5 M$ ). Changes in neuronal morphology in adult APP/PS1 mice after immune stimulation with LPS were less dramatic than in aged APP/PS1. However, the adult AD mice show a stronger phenotype than their adult WT counterparts making them more sensitive to the additional inflammatory challenge with LPS.

The long-term consequence of a peripheral immune stimulation with LPS on neuronal function in the hippocampal circuitry was examined in aged APP/PS1 mice. LTP was successfully induced and maintained stable under all experimental conditions. The induction and maintenance of LTP was significantly lower three months after treatment with both LPS-types from $E$. coli and from S. typhimurium when compared with controlinjected APP/PS1 mice (APP/PS1 19 months mean LTP two-way ANOVA $F_{(2,56)}=5.535 ; p=0.0064 ; \mathrm{APP} / \mathrm{PS} 119$ months last 10 min LTP one-way ANOVA $F_{(2,56)}=5.806 ; p=0.0051$ : LPS E. coli Fisher's LSD $p=0.0045$; LPS S. typhimurium Fisher's LSD $p=0.0061$; Fig. $5 F$ ). Basal synaptic transmission was not altered in the LPS S. typhimurium-treated group, but surprisingly, in slices from LPS E. coli-treated old APP/PS1 mice, the fEPSP slope was significantly higher than control signals (APP/PS1 19 months I/O two-way ANOVA $F_{(2,60)}=4.567 ; p=0.0143$ : LPS E. coli Turkey's 200-600 $\mu \mathrm{A} p<0.025$; Fig. 5G). Values of the PPF paradigm were on control level with both types of LPS (APP/PS1 19 months PPF two-way ANOVA $F_{(2,41)}=0.7965$; $p=0.4578$; Fig. $5 H$ ). Taken together, these data support the view that APP/PS1 mice are more susceptible to an additional systemic inflammatory insult.

\section{The detrimental effects of LPS S. typhimurium in aged WT mice are NLRP3 inflammasome dependent}

As the absence of the NLRP3 inflammasome was shown to rescue the neurodegenerative phenotype seen in APP/PS1 mice 


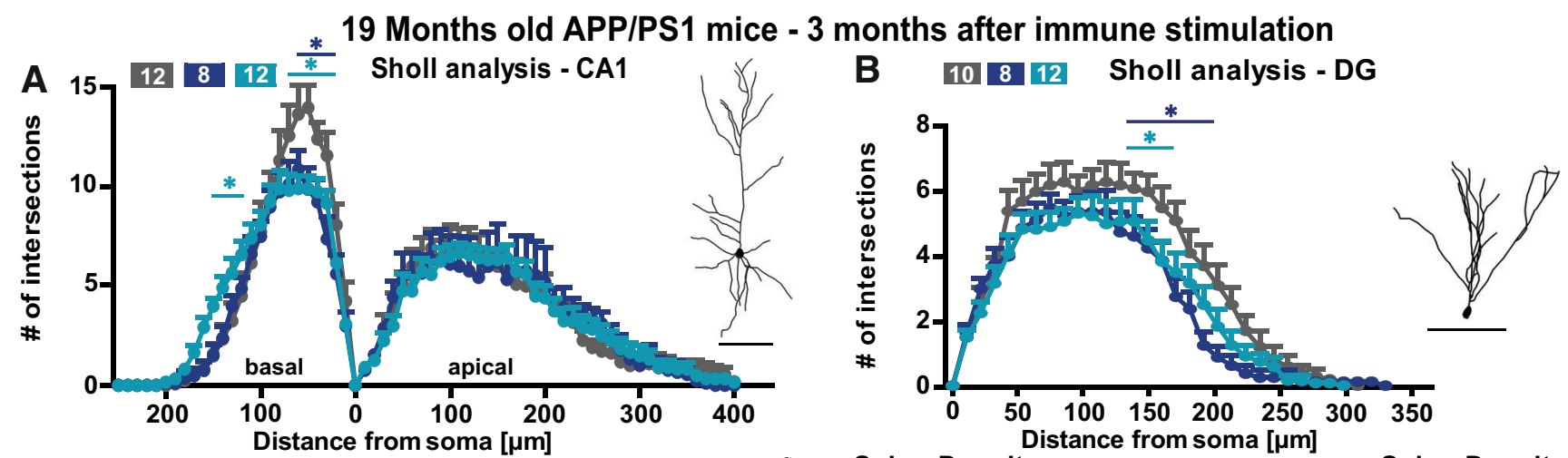

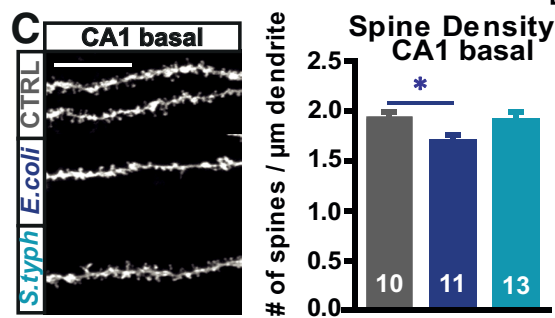

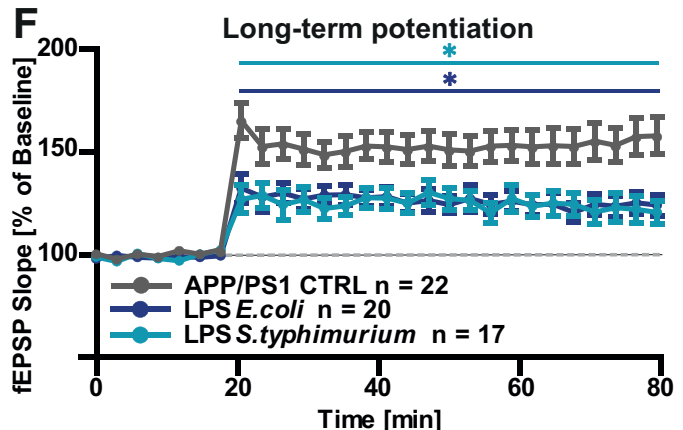

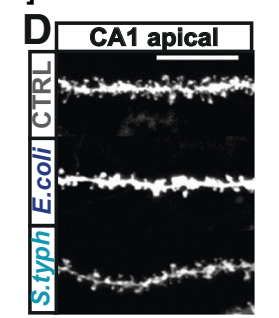

Mean LTP $70-80 \mathrm{~min}$

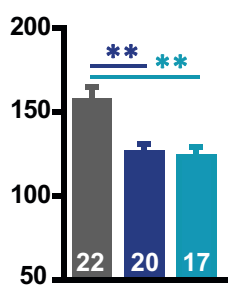

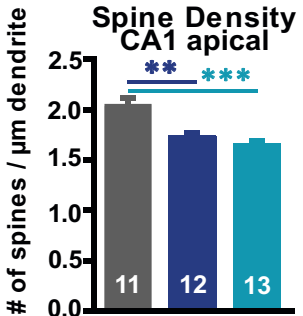
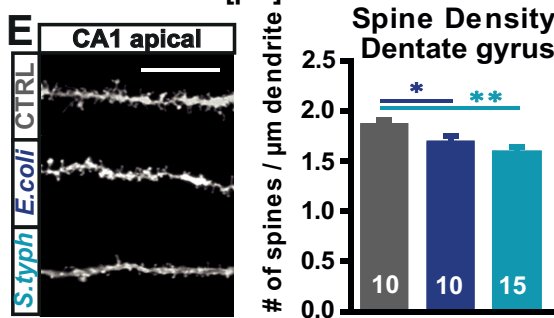

G Basal synaptic transmis- H Paired pulse facilitation
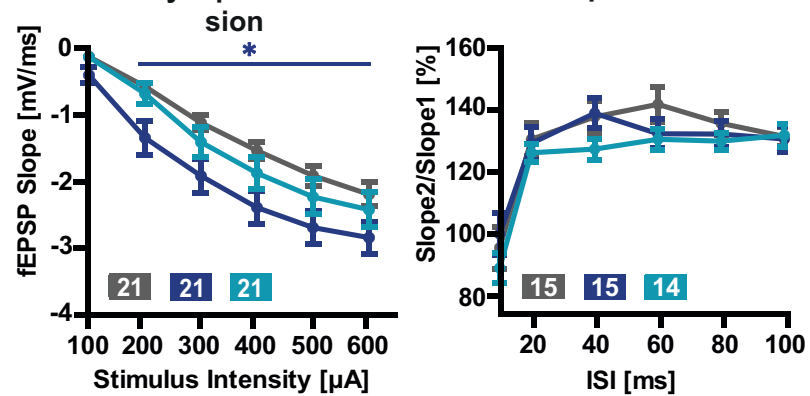

7 Months old APP/PS1 mice - 3 months after immune stimulation
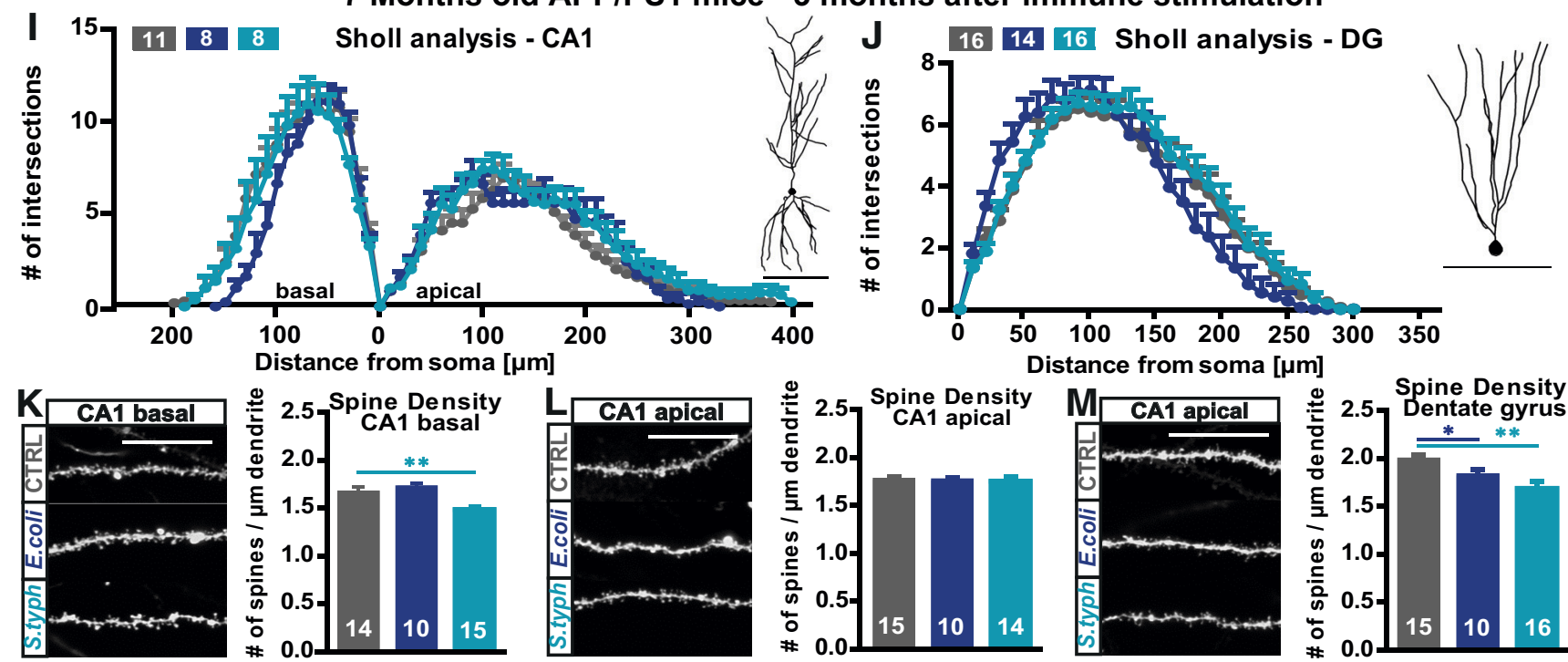

APP/PS1 CTRL

LPS E.coli

LPS S.typhimurium

Figure 5. Long-term effects of LPS on neuronal morphology and synaptic plasticity in adult and aged APP/PS1 mice. A-E, Neuronal architecture of 19-month-old APP/PS1 mice, CTRL (gray), LPS E. coli $(0.4 \mu \mathrm{g} / \mathrm{g}$ bodyweight, dark blue), and LPS S. typhimurium ( $0.4 \mu \mathrm{g} / \mathrm{g}$ bodyweight, light blue): Sholl analysis and typical tracing of (A) CA1 pyramidal neurons and (B) granule cells of the DG. Number of spines per $\mu \mathrm{m}$ and confocal microscopic image of Dil-stained ( $\boldsymbol{C}$ basal CA1 dendrites, (D) mid-apical CA1 dendrites, and (E) DG granule cells; $N=3, n=8-15$. $\boldsymbol{F}-\boldsymbol{H}$, Changes in shortterm and long-term synaptic plasticity in the hippocampus of 19-month-old APP/PS1 mice three months after peripheral immune stimulation: $(\boldsymbol{F})$ LTP at Schaffer collateral pathway measured at stimulus intensity of 40\% maximal fEPSP slope, LTP induction after 20 min of baseline recording via TBS, mean LTP as fEPSP slope \% of baseline of the last 10 min of recording (minutes $70-80$ ). $\boldsymbol{G}$, Input-out-relationship, fEPSP slope measured at increasing stimulus intensities. $\boldsymbol{H}$, PPF at $40 \%$ of the maximal fEPSP slope; $n=$ number of hippocampal acute slices; $N=3-6, n=11-22$. $I-M$, Neuronal morphology of seven-month-old APP/PS1 mice, CTRL (gray), LPS E. coli (dark blue), and LPS S. typhimurium (light blue): Sholl analysis and tracing of (I) CA1 pyramidal neurons and ( $\boldsymbol{J}$ granule cells of the DG. Spine density and representative confocal microscopic images of (K) basal CA1 dendrites, (L) mid-apical CA1 dendrites, and $(\boldsymbol{M})$ of DG granule cells. Tracing scale bars, $100 \mu \mathrm{m}$; scale bar for confocal microscopic images, $10 \mu \mathrm{m} ; n=$ number of cells; $N=3, n=10-15$ all data are presented as mean \pm SEM, ${ }^{*} p<0.05,{ }^{* *} p<0.01 ;{ }^{* * *} p<0.001$. 
(Heneka et al., 2013), we hypothesized that it could also be beneficial in the context of a peripheral immune stimulation with LPS of $S$. typhimurium in aged mice. Therefore, 16-month-old NLRP3 KO mice were immune stimulated via peripheral administration of S. typhimurium LPS, and neuronal architecture as well as synaptic plasticity at the Schaffer collaterals were analyzed three months later. Indeed, Sholl analysis and quantification of the spine density of CA1 and DG neurons show neuronal morphology of immune-stimulated NLRP3 KO mice on control levels (NLRP3 KO 19 months CA1 basal two-way ANOVA $F_{(1,31)}=$ 3.032; $p=0.0916$ : LPS S. typhimurium Fisher's LSD distance 40$100 \mu \mathrm{m} p<0.015$; CA1 apical two-way ANOVA $F_{(1,28)}=1.402$; $p=0.2463$ : LPS $S$. typhimurium Fisher's LSD distance 220-230 $\mu \mathrm{m} \quad p<0.048$; DG two-way ANOVA $F_{(1,33)}=0.04,838$; $p=0.8273$; Fig. $6 A-E)$. Analysis of synaptic plasticity revealed that the NLRP3 KO fully protected aged mice from S. typhimurium LPS-induced LTP suppression (NLRP3 KO 19 months mean LTP two-way ANOVA $F_{(1,38)}=0.1337 ; p=0.7167$; NLRP3 KO 19 months last 10 min LTP unpaired $t$ test $t=0.4395 \mathrm{df}=38$; $p=0.6628$ ), which was shown in aged WT animals (Fig. 6I, also see Fig. $4 A-C$; mean LTP two-way ANOBA $F_{(1,31)}=8.512$; $p=0.0065$; last $10 \mathrm{~min}$ LTP unpaired $t$ test $t=2.456 \mathrm{df}=31$; $p=0.0198$; Fig. $6 F$ ). Also, in these mice, the basal synaptic transmission within the CA3-CA1 pathway was not affected by $S$. typhimurium LPS (Fig. 6G,J). The presynaptic properties were not affected either as there was no change in the PPF paradigm (Fig. 6H,K).

Taken together, the lack of NLRP3 prevented detrimental effects on synaptic plasticity and neuronal architecture caused by peripheral immune stimulation with S. typhimurium LPS in aged mice. This might be due to a decreased activation of microglia. While the number of microglia is significantly increased by $S$. typhimurium LPS in aged NLRP3 KO as well as WT mice three months after immune stimulation (NLRP3 KO 19 months cell number unpaired $t$ test $t=2.097 \mathrm{df}=19 ; p=0.0496$; WT 19 months cell number $t=3.324 \mathrm{df}=27 ; p=0.0026$; Fig. $6 M$ ), the strong activation of microglia, quantified by a decreased number of primary processes, as seen in WT mice, is absent in NLRP3 KO mice (NLRP3 KO 19 months primary processes unpaired $t=0.6535 \mathrm{df}=19 ; p=0.6535$; WT 19 months primary processes $t=6.827 \mathrm{df}=26 ; p<0.0001$; Fig. $6 N)$.

Hypothesizing a long-lasting activation of the NLRP3 inflammasome (Christ et al., 2018) due to LPS injection, we further analyzed whether also the acute inhibition of the NLRP3 inflammasome after the immune stimulation and the formation of neuroinflammation is beneficial for neuronal morphology and function in aged mice. Therefore, 16-month-old C57Bl/6J WT mice were peripherally immune stimulated with $S$. typhimurium LPS as before. After three months, the small NLRP3 inhibitor MCC950 (Coll et al., 2015) was applied via intraperitoneal injection for two consecutive days directly before the analysis of neuronal architecture and synaptic plasticity. The dendritic complexity as well as spine density of CA1 pyramidal neurons and DG granule cells were on control levels or even increased despite the immune stimulation with $S$. typhimurium LPS (WT 19 months MCC950 CA1 basal two-way ANOVA $F_{(1,27)}=$ 0.4138; $p=0.5255$ : LPS $S$. typhimurium Fisher's LSD distance $100-120 \mu \mathrm{m} p<0.044$; CA1 apical two-way ANOVA $F_{(1,26)}=$ $0.7709 ; p=0.3880$ : LPS $S$. typhimurium Fisher's LSD distance 70-130 $\mu \mathrm{m} p<0.044$; DG two-way ANOVA $F_{(1,27)}=0.01,674$; $p=0.8980$; Fig. $7 A-E$ ). Likewise, also the magnitude of LTP at the CA3-CA1 pathway was comparable between immune-stimulated and control mice (WT 19 months MCC950 mean LTP:
CTRL $139.8 \pm 9.1$; LPS S. typhimurium $140.9 \pm 8.1, p=0.9289$; Fig. $7 F)$. Interestingly, also the number of microglia cells was on control level after NLRP3 inflammasome inhibition, and microglia activation was at least diminished (WT 19 months MCC950 cell number: CTRL $1.00 \pm 0.10$; LPS S. typhimurium $0.88 \pm 0.07$, $p=0.2942$; primary processes: CTRL $7.81 \pm 0.48$; LPS S. typhimurium $6.63 \pm 0.23, p=0.0378$; Fig. $7 J, K)$. In a control experiment, MCC950 was applied to aged WT mice which were not immune stimulated via LPS S. typhimurium to quantify possible side and off target effects in the aged mouse brain. Here, no differences in synaptic plasticity between the MCC950-treated and control group were visible (Fig. $7 L-N$ ).

The NLRP3 inflammasome therefore seems to be an important player for persisting cerebral changes after systemic inflammation.

\section{Discussion}

In the case of sepsis-induced encephalopathy, peripheral inflammation can cause acute cerebral dysfunction without direct CNS injury or infection (Pine et al., 1983; Sprung et al., 1990; Wilson and Young, 2003; Widmann and Heneka, 2014). In rodent models, an immune challenge with peripheral administration of LPS, a component of the Gram-negative bacterial cell wall, can mimic sepsis-induced impairments in cognition and memory as it increases the production of brain proinflammatory cytokines and immune cell activation in the brain which leads to learning and memory deficits in certain behavioral tasks and impairs LTP in an acute manner (Arai et al., 2001; Commins et al., 2001; Jo et al., 2001; Hennigan et al., 2007; Semmler et al., 2007; Cunningham et al., 2009; Di Filippo et al., 2013; Strehl et al., 2014). However, long-term effects of a stimulation of the peripheral immune system on neuronal structure and function are highly unexplored.

We hypothesized that peripheral immune stimulation can cause persisting dysfunction of learning and memory processes, in particular when brain immune cells are already in a "primed state" as found with age and in mouse models of AD. Indeed, we are able to demonstrate that aged mice exhibit a decrease in neuronal complexity and spine density of hippocampal neurons whereas adult mice do not show any overt changes three months after a peripheral immune stimulation with LPS. We further observed that the type of LPS is relevant for the magnitude of decline. While alterations in neuronal architecture are visible with LPS from E. coli as well as $S$. typhimurium, impairment in LTP three months after immune stimulation is only detectable with S. typhimurium LPS. Also, disturbance of spatial learning in the Morris water maze task is only obvious with this unconventional LPS. Explanation for this might be the different strength of peripheral immune cell or local microglia/macrophage activation. LPS of $S$. typhimurium results in a higher number of microglia and a stronger activation than E. coli LPS. Since the exposure to bacteria and systemic inflammation is a frequent event in an aging population, our study helps to further improve modeling of neurodegenerative disease in murine models.

In rodents, about half of the genes upregulated with age are associated with inflammation and oxidative stress (Prolla, 2002). Also, in our hands, aged mice show a higher activation of microglia already under basal conditions. A similar outcome was shown in a CD200-deficient mouse stimulated with LPS, where a hyperactivation of microglia impaired LTP (Costello et al., 2011). In line with this, inhibition of microglia rescues LTP deficits induced by chronic systemic inflammation seen in middle aged 


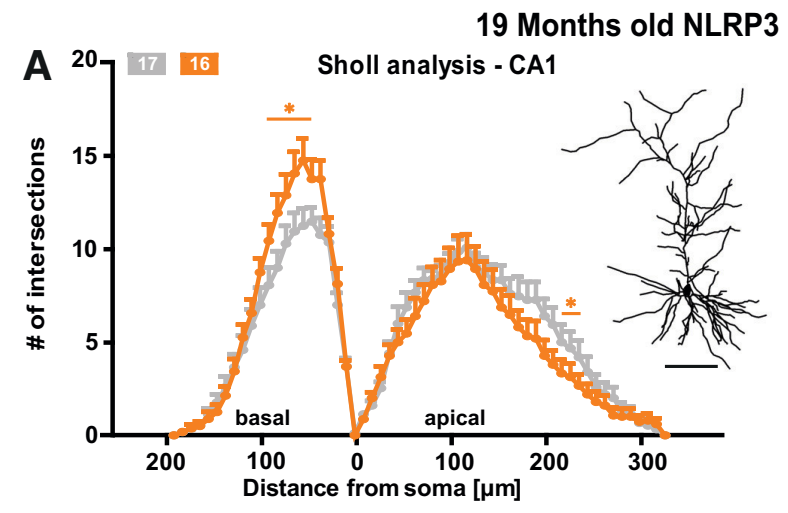
B 17 Sholl analysis - DG

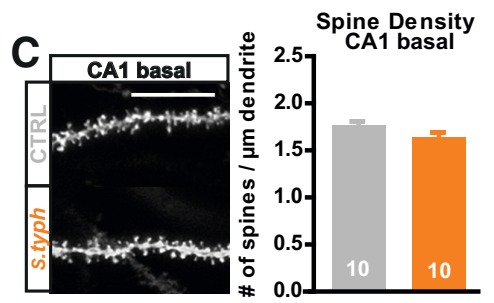

F Long-term potentiation
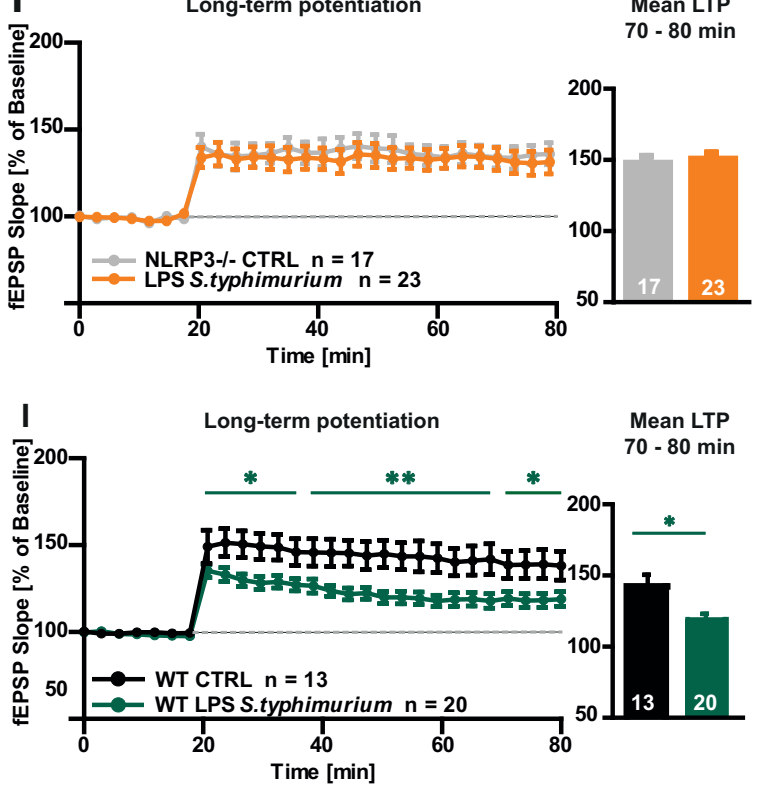
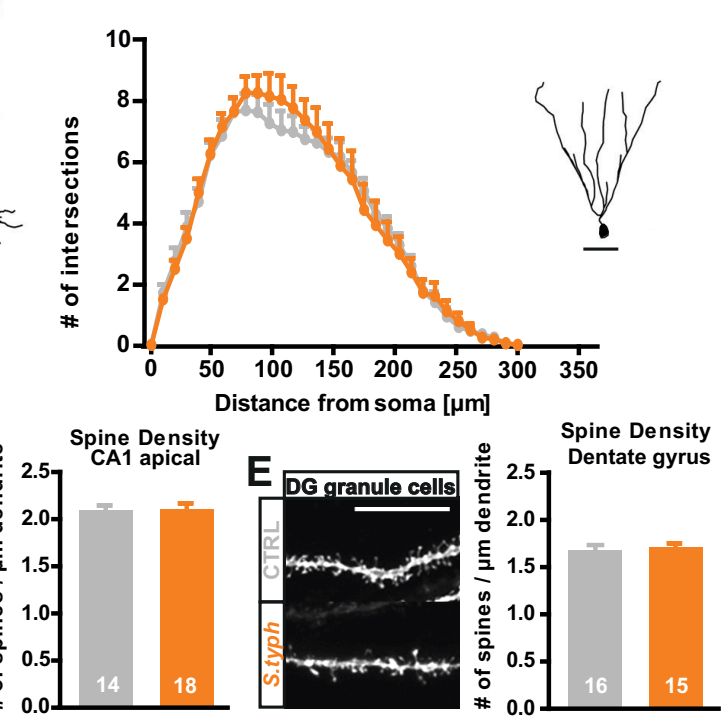

G Basal synaptic transmission $H$ Paired pulse facilitation
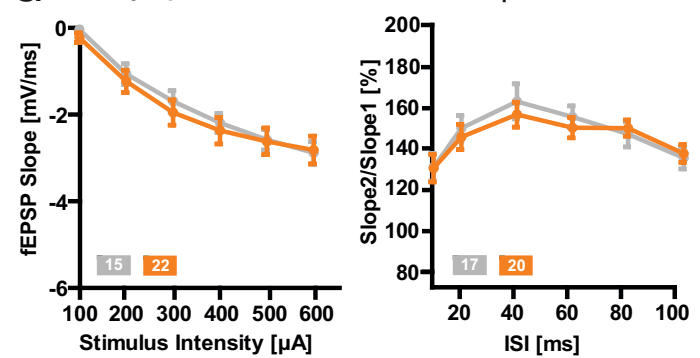

$\mathbf{J}$ Basal synaptic transmission $\mathbf{K}$
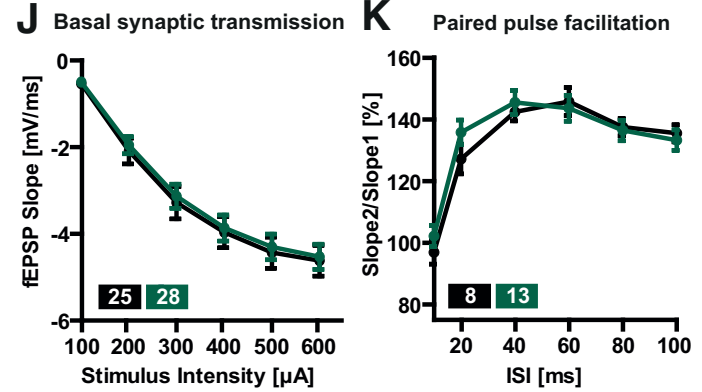
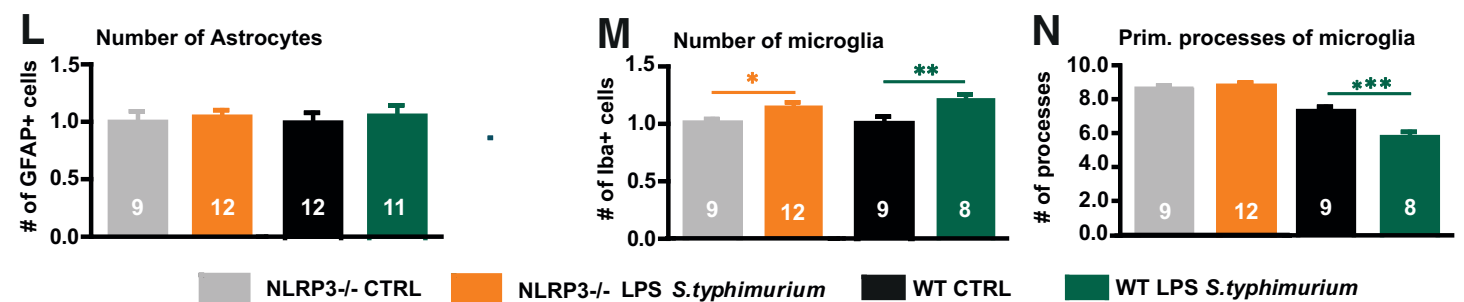

NLRP3-/- LPS S.typhimurium

WT CTRL

WT LPS S.typhimurium

Figure 6. Neuronal morphology and synaptic plasticity of aged NLRP3 KO mice after immune stimulation with S. typhimurium LPS. A-E, Neuronal architecture of 19-month-old NLRP3 KO mice; CTRL (gray), LPS S. typhimurium (orange): tracings and Sholl analysis of (A) CA1 pyramidal neurons and (B) DG granule cells. Spine density and exemplified confocal microscopic images from (D) mid-apical CA1 dendrites, $(\boldsymbol{C})$ CA1 basal dendrites, and $(\boldsymbol{E})$ of granule cells from the DG. $\boldsymbol{F}$ - $\boldsymbol{H}$, Short-term and long-term synaptic plasticity at the CA3-CA1 pathway in hippocampal acute slices of aged NLRP3 K0 mice three months after peripheral immune stimulation: $(\boldsymbol{F})$ LTP induced via TBS shown as fEPSP slope \% of baseline and mean LTP as fEPSP slope \% of baseline for minutes $70-80$ of recording. $\boldsymbol{G}$, Input-output curve. $\boldsymbol{H}$, PPF with increasing ISIs. I- $\boldsymbol{K}$, Short-term and long-term synaptic plasticity at the CA3-CA1 pathway in hippocampal acute slices of aged WT mice three months after peripheral immune stimulation: (I) LTP induced via TBS shown as fEPSP slope $\%$ of baseline and mean LTP as fEPSP slope \% of baseline for minutes 70-80 of recording. J, Input-output curve. $\boldsymbol{K}$, PPF with increasing ISIs. $\mathbf{L}-\mathbf{N}$, Astrocyte and microglia phenotype in the stratum radiatum of the CA1 area of aged NLRP3 K0 mice three months after peripheral immune stimulation: (L) quantification of GFAP-positive cells in aged NLRP3 KO mice (CTLR gray, LPS S. typhimurium orange) and WT mice (CTRL black, LPS S. typhimurium green). $\boldsymbol{M}$, Relative number of IBA-1-positive cells of immune-stimulated mice compared with the respective aged controls, NLRP3 K0 mice (gray, orange), WT mice (black, green) and the change between both immune-stimulated groups in \%. $\mathbf{N}$, Number of primary branches of IBA-1-positive cells in control (gray) and immune-stimulated aged NLRP3 K0 mice (orange) and immune-stimulated WT mice with (black, green) and comparison between the immune-stimulated groups as fold change, respectively; $N=3-4$ mice per group, $n=9-23$ number of samples; scale bar of tracings, $100 \mu \mathrm{m}$; scale bar of confocal fluorescent images, $10 \mu \mathrm{m}$; all data are presented as mean $\pm \mathrm{SEM} ;{ }^{*} p<0.05,{ }^{* *} p<0.01 ;{ }^{* * *} p<0.001$. 

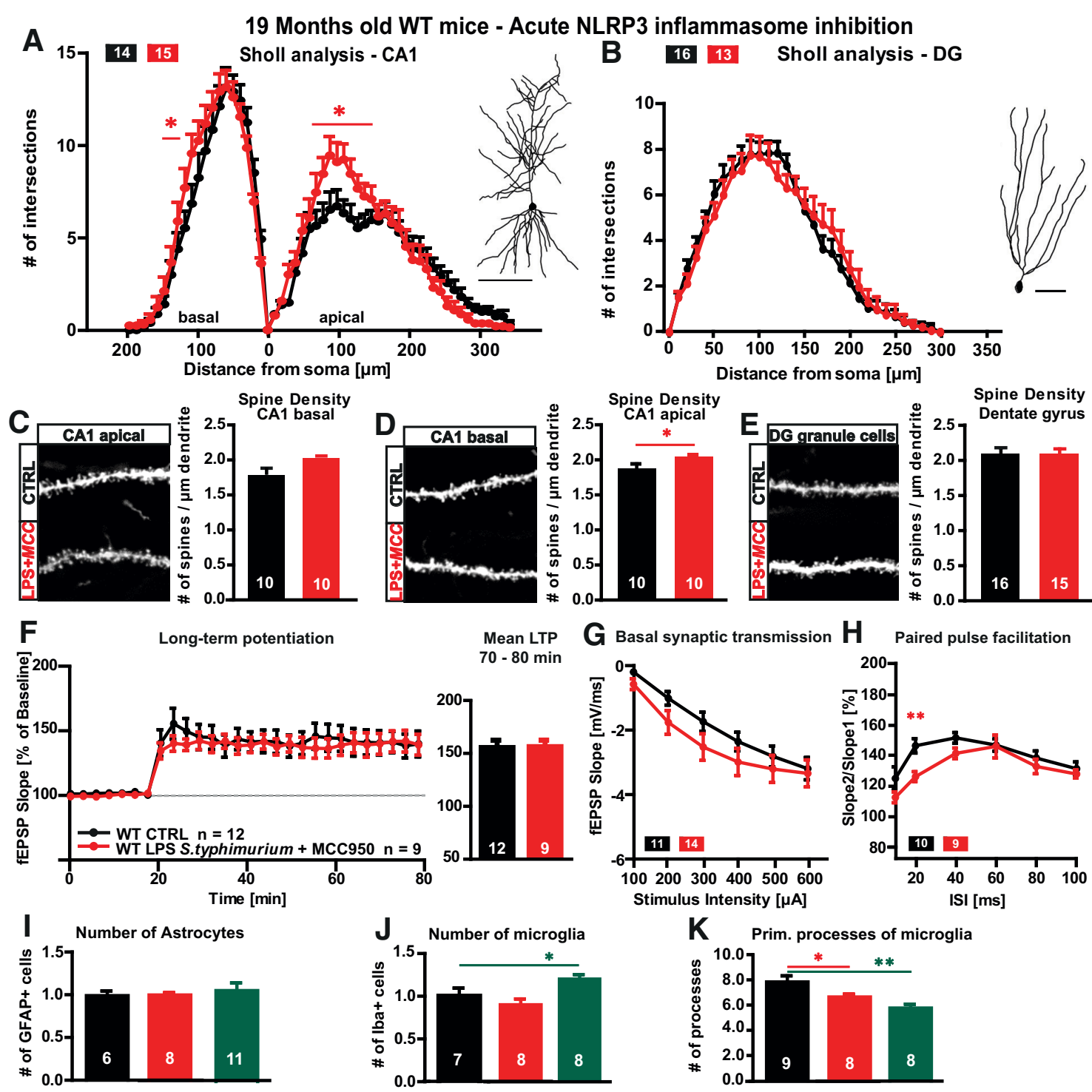

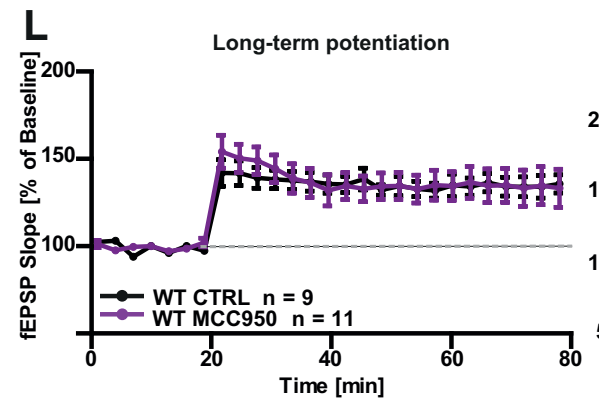

WT CTRL

WT LPS S.typhimurium

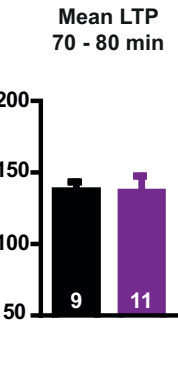

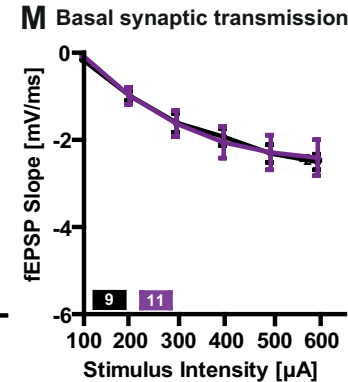

Stimulus Intensity $[\mu \mathrm{A}]$

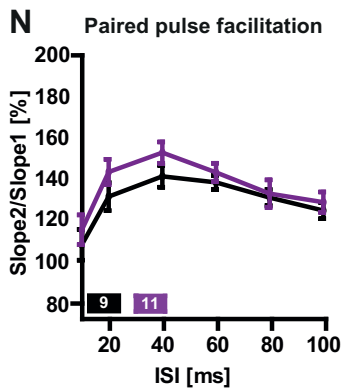

WT LPS S.typhimurium + MCC950

WT CTRL MCC950

Figure 7. Neuronal morphology and synaptic plasticity of aged LPS immune-stimulated WT mice after MCC950 treatment $\boldsymbol{A}-\boldsymbol{E}$, Neuronal architecture of 19-month-old WT mice, (TRL (black) versus LPS S. typhimurium treated with MCC $950(20 \mu \mathrm{g} / \mathrm{g}$ bodyweight, red): dendritic complexity and exemplified tracing of $(\boldsymbol{A})$ CA1 hippocampal neurons and $(\boldsymbol{B})$ DG granule cells. Confocal microscopic image and spine density of $(\boldsymbol{C})$ basal CA1 dendrites, $(\boldsymbol{D})$ mid-apical CA1 dendrites, and (E) DG granule cells. $\boldsymbol{F}-\boldsymbol{H}$, Synaptic plasticity of aged WT mice, CTRL versus LPS S. typhimurium treated with MCC950: (F) LTP as fEPSP slope \% of baseline and quantification of 70-80 min of LTP measurement. G, Input-out properties. $\boldsymbol{H}$, Short-term synaptic plasticity, PPF. I- $\boldsymbol{K}$, Astrocyte and microglia phenotype in the stratum radiatum of the CA1 area of aged MCC950-treated WT mice three months after peripheral immune stimulation: (I) relative number of GFAP-positive cells: CTRL versus LPS S. typhimurium with MCC950 treatment (red) and CTRL (black) versus LPS S. typhimurium (green). J, Quantification of IBA-1-positive cells in WT mice, CTRL (black) versus LPS S. typhimurium with (red), or without MCC950 treatment (green), differences between both immune-stimulated groups as fold change in \%. $\boldsymbol{K}$, Number of primary branches of IBA-1-positive cells in controls (black) and immune-stimulated WT mice with (red) or without MCC950 (green) and comparison between the immune-stimulated groups as fold change, respectively. $L-N$, Synaptic plasticity of aged WT mice, CTRL versus CTRL treated with MCC950: (L) LTP as fEPSP slope \% of baseline and quantification of 70-80 min of LTP measurement. $M$, Input-out properties. $\boldsymbol{N}$, Short-term synaptic plasticity, PPF; $N=3$ mice per group, $n=7-15$ number of samples; scale bar of tracings, $100 \mu \mathrm{m}$; scale bar of confocal fluorescent images, $10 \mu \mathrm{m}$; all data are presented as mean $\pm \mathrm{SEM} ;{ }^{*} p<0.05,{ }^{* *} p<0.01$. 
rats, whereas young rats do not show any LTP impairment in this model (Liu et al., 2012). The expression of proinflammatory cytokines is increased in aged mice and immune stimulation with LPS enhances this production even further (Chen et al., 2008).

Like in aged individuals, also the brains of $\mathrm{AD}$ mice are in an already challenged condition (Glass et al., 2010). We saw an even higher vulnerability of aged APP/PS1 mice toward a systemic inflammation and impaired LTP with S. typhimurium as well as E. coli LPS. Already younger AD mice show negative effects on neuronal architecture on immune stimulation. And again, these mice are not protected from LPS of E. coli.

To our knowledge, only one study ever showed a difference in the inflammatory signaling on recognition of LPS from E. coli and S. typhimurium. Here, S. typhimurium LPS induces higher concentrations of IL- $1 \beta$ and IFN- $\gamma$ in a TNF $\alpha$-independent way. In contrast, response to E. coli LPS involves TNF $\alpha$ and leads to lower IL- $1 \beta$ and IFN- $\gamma$ production (Netea et al., 2001). Only $6 \mathrm{~h}$ after immune stimulation, we saw a strong increase in IL-6 and IL- $1 \beta$ in blood plasma with both LPS types, but higher concentrations of IL- $1 \beta$ in the brains of S. typhimurium LPS-treated mice when compared with E. coli LPS fitting the above-mentioned study.

Therefore, we checked whether the neurodegenerative phenotype seen in aged WT mice on LPS S. typhimurium treatment can be rescued by a genetic NLRP3 inflammasome KO or pharmacological inhibition. Recent published data showed that NLRP3 KO mice are persistent against peripheral immune challenge and showed no morphologic changes in microglia phenotype (Tejera et al., 2019). Loss of the NLRP3 inflammasome can improve LTP and cognitive dysfunction observed in APP/PS1 mice in the absence of a peripheral immune challenge (Heneka et al., 2013). Here, it is important to note that NLRP3 inflammasome-generated cytokines such as IL-1 $\beta$ and Il-18 have been repeatedly shown to negatively impact on synaptic plasticity (Curran and O'Connor, 2001; Pickering and O'Connor, 2007; Lynch, 2015). In the present study, immune challenged mice are protected from alterations of neuronal morphology and dysfunction on genetic deletion of NLRP3, indicating that downstream effectors of NLRP3 inflammasome activation, including IL- $1 \beta$ and IL-18, contribute to the sustained cerebral changes in response to systemic immune stimulation. Of note, not only genetic ablation of NLRP3 but acute pharmacological inhibition by the NLRP3 inhibitor MCC950 rescued the neurodegenerative phenotype observed in aged WT mice. Moreover, when S. typhimurium LPS-stimulated mice were treated with MCC950 for only $2 \mathrm{~d}$, neuronal morphology and function were indistinguishable from control mice. This neuroprotective phenomenon was associated with a profound decrease of microglia number and activation in comparison to untreated mice, which showed a long-lasting activation of microglia cells, comparable to longlasting consequences of an LPS-independent stimulus (Western diet), which also showed a long-lasting activation of microglia cells (Christ et al., 2018).

The present study provides new evidence for an age- and NLRP3-dependent cerebral vulnerability toward a systemic immune challenge with $S$. typhimurium LPS. The precise site of interference with the NLRP3 inflammasome, locally in microglia/macrophages or alternatively in peripheral immune cells, cannot be answered. However, the positive effects of pharmacological NLRP3 inhibition on brain structure and function opens new avenues for preventing long-term brain dysfunction after systemic inflammatory events such as a severe systemic bacterial infection.

\section{References}

Angus DC, Linde-Zwirble WT, Lidicker J, Clermont G, Carcillo J, Pinsky MR (2001) Epidemiology of severe sepsis in the United States: analysis of incidence, outcome, and associated costs of care. Crit Care Med 29:13031310.

Arai K, Matsuki N, Ikegaya Y, Nishiyama N (2001) Deterioration of spatial learning performances in lipopolysaccharide-treated mice. Jpn J Pharmacol 87:195-201.

Biesmans S, Meert TF, Bouwknecht JA, Acton PD, Davoodi N, De Haes P, Kuijlaars J, Langlois X, Matthews LJR, Ver Donck L, Hellings N, Nuydens R (2013) Systemic immune activation leads to neuroinflammation and sickness behavior in mice. Mediators Inflamm 2013:271359.

Bone RC (1992) Toward an epidemiology and natural history of SIRS (systemic inflammatory response syndrome). JAMA 268:3452-3455.

Borchelt DR, Ratovitski T, Van Lare J, Lee MK, Gonzales V, Jenkins NA, Copeland NG, Price DL, Sisodia SS (1997) Accelerated amyloid deposition in the brains of transgenic mice coexpressing mutant presenilin 1 and amyloid precursor proteins. Neuron 19:939-945.

Cannon JG, Tompkins RG, Gelfand JA, Michie HR, Stanford GG, Van Der Meer JW, Endres S, Lonnemann G, Corsetti J, Chernow B (1990) Circulating interleukin-1 and tumor necrosis factor in septic shock and experimental endotoxin fever. J Infect Dis 161:79-84.

Chen J, Buchanan JB, Sparkman NL, Godbout JP, Freund GG, Johnson RW (2008) Neuroinflammation and disruption in working memory in aged mice after acute stimulation of the peripheral innate immune system. Brain Behav Immun 22:301-311.

Christ A, Günther P, Lauterbach MA, Duewell P, Biswas D, Pelka K, Scholz CJ, Oosting M, Haendler K, Baßler K, Klee K, Schulte-Schrepping J, Ulas T, Moorlag SJ, Kumar V, Park MH, Joosten LA, Groh LA, Riksen NP, Espevik T, et al. (2018) Western diet triggers NLRP3-dependent innate immune reprogramming. Cell 172:162-175.e14.

Coll RC, Robertson AA, Chae JJ, Higgins SC, Muñoz-Planillo R, Inserra MC, Vetter I, Dungan LS, Monks BG, Stutz A, Croker DE, Butler MS, Haneklaus M, Sutton CE, Núñez G, Latz E, Kastner DL, Mills KH, Masters SL, et al. (2015) A small-molecule inhibitor of the NLRP3 inflammasome for the treatment of inflammatory diseases. Nat Med 21:248255.

Commins S, O'Neill LA, O'Mara SM (2001) The effects of the bacterial endotoxin lipopolysaccharide on synaptic transmission and plasticity in the CA1-subiculum pathway in vivo. Neuroscience 102:273-280.

Costello DA, Lyons A, Denieffe S, Browne TC, Cox FF, Lynch MA (2011) Long term potentiation is impaired in membrane glycoprotein CD200deficient mice a role for toll-like receptor activation. J Biol Chem 286:34722-34732.

Cunningham C, Campion S, Lunnon K, Murray CL, Woods JF, Deacon RM, Rawlins JNP, Perry VH (2009) Systemic inflammation induces acute behavioral and cognitive changes and accelerates neurodegenerative disease. Biol Psychiatry 65:304-312.

Curran B, O'Connor J (2001) The pro-inflammatory cytokine interleukin-18 impairs long-term potentiation and NMDA receptor-mediated transmission in the rat hippocampus in vitro. Neuroscience 108:83-90.

DiCarlo G, Wilcock D, Henderson D, Gordon M, Morgan D (2001) Intrahippocampal LPS injections reduce Abeta load in APP+ PS1 transgenic mice. Neurobiol Aging 22:1007-1012.

Di Filippo M, Chiasserini D, Gardoni F, Viviani B, Tozzi A, Giampà C, Costa C, Tantucci M, Zianni E, Boraso M, Siliquini S, de Iure A, Ghiglieri V, Colcelli E, Baker D, Sarchielli P, Fusco FR, Di Luca M, Calabresi P (2013) Effects of central and peripheral inflammation on hippocampal synaptic plasticity. Neurobiol Dis 52:229-236.

Garthe A, Behr J, Kempermann G (2009) Adult-generated hippocampal neurons allow the flexible use of spatially precise learning strategies. PLoS One 4:e5464.

Glass CK, Saijo K, Winner B, Marchetto MC, Gage FH (2010) Mechanisms underlying inflammation in neurodegeneration. Cell 140:918-934.

Godbout J, Chen J, Abraham J, Richwine A, Berg B, Kelley K, Johnson RW (2005) Exaggerated neuroinflammation and sickness behavior in aged mice following activation of the peripheral innate immune system. FASEB J 19:1329-1331.

Halle A, Hornung V, Petzold GC, Stewart CR, Monks BG, Reinheckel T, Fitzgerald KA, Latz E, Moore KJ, Golenbock DT (2008) The NALP3 inflammasome is involved in the innate immune response to amyloid- $\beta$. Nat Immunol 9:857-865 
Heneka MT, Kummer MP, Stutz A, Delekate A, Schwartz S, Vieira-Saecker A, Griep A, Axt D, Remus A, Tzeng T-C, Gelpi E, Halle A, Korte M, Latz E, Golenbock DT (2013) NLRP3 is activated in Alzheimer's disease and contributes to pathology in APP/PS1 mice. Nature 493:674-678.

Heneka MT, Golenbock DT, Latz E (2015) Innate immunity in Alzheimer's disease. Nat Immunol 16:229-236.

Heneka MT, McManus RM, Latz E (2018) Inflammasome signalling in brain function and neurodegenerative disease. Nat Rev Neurosci 19:610-621.

Hennigan A, Trotter C, Kelly ÁM (2007) Lipopolysaccharide impairs longterm potentiation and recognition memory and increases p75NTR expression in the rat dentate gyrus. Brain Res 1130:158-166.

Holmes C, Butchart J (2011) Systemic inflammation and Alzheimer's disease. London: Portland Press Ltd.

Holmes C, Cunningham C, Zotova E, Woolford J, Dean C, Kerr S, Culliford D, Perry V (2009) Systemic inflammation and disease progression in Alzheimer disease. Neurology 73:768-774.

Iwashyna TJ, Ely EW, Smith DM, Langa KM (2010) Long-term cognitive impairment and functional disability among survivors of severe sepsis. JAMA 304:1787-1794.

Jankowsky JL, Fadale DJ, Anderson J, Xu GM, Gonzales V, Jenkins NA, Copeland NG, Lee MK, Younkin LH, Wagner SL, Younkin SG, Borchelt DR (2004a) Mutant presenilins specifically elevate the levels of the 42 residue $\beta$-amyloid peptide in vivo: evidence for augmentation of a 42-specific $\gamma$ secretase. Hum Mol Genet 13:159-170.

Jankowsky JL, Slunt HH, Gonzales V, Jenkins NA, Copeland NG, Borchelt DR (2004b) APP processing and amyloid deposition in mice haplo-insufficient for presenilin 1. Neurobiol Aging 25:885-892.

Jo JH, Park EJ, Lee JK, Jung MW, Lee CJ (2001) Lipopolysaccharide inhibits induction of long-term potentiation and depression in the rat hippocampal CA1 area. Eur J Pharmacol 422:69-76.

Kummer MP, Hermes M, Delekarte A, Hammerschmidt T, Kumar S, Terwel D, Walter J, Pape H-C, König S, Roeber S, Jessen F, Klockgether T, Korte M, Heneka MT (2011) Nitration of tyrosine 10 critically enhances amyloid $\beta$ aggregation and plaque formation. Neuron 71:833-844.

Latz E (2010) The inflammasomes: mechanisms of activation and function. Curr Opin Immunol 22:28-33.

Lee DC, Rizer J, Selenica M-L, Reid P, Kraft C, Johnson A, Blair L, Gordon MN, Dickey CA, Morgan D (2010) LPS-induced inflammation exacerbates phospho-tau pathology in rTg4510 mice. J Neuroinflammation 7:56.

Letiembre M, Hao W, Liu Y, Walter S, Mihaljevic I, Rivest S, Hartmann T, Fassbender K (2007) Innate immune receptor expression in normal brain aging. Neuroscience 146:248-254.

Liu X, Wu Z, Hayashi Y, Nakanishi H (2012) Age-dependent neuroinflammatory responses and deficits in long-term potentiation in the hippocampus during systemic inflammation. Neuroscience 216:133-142.

Lynch MA (2015) Neuroinflammatory changes negatively impact on LTP: a focus on IL-1 $\beta$. Brain Res 1621:197-204.

Michaud JP, Hallé M, Lampron A, Thériault P, Préfontaine P, Filali M, Tribout-Jover P, Lanteigne AM, Jodoin R, Cluff C, Brichard V, Palmantier R, Pilorget A, Larocque D, Rivest S (2013) Toll-like receptor 4 stimulation with the detoxified ligand monophosphoryl lipid A improves Alzheimer's disease-related pathology. Proc Natl Acad Sci USA 110:1941-1946.

Michie HR, Manogue KR, Spriggs DR, Revhaug A, O’Dwyer S, Dinarello CA, Cerami A, Wolff SM, Wilmore DW (1988) Detection of circulating tumor necrosis factor after endotoxin administration. N Engl J Med 318:1481-1486.

Morris R (1984) Developments of a water-maze procedure for studying spatial learning in the rat. J Neurosci Methods 11:47-60.

Netea MG, Kullberg BJ, Joosten LA, Sprong T, Verschueren I, Boerman OC, Amiot F, van den Berg WB, Van der Meer JW (2001) Lethal Escherichia coli and Salmonella typhimurium endotoxemia is mediated through different pathways. Eur J Immunol 31:2529-2538.

Nguyen MD, Julien J-P, Rivest S (2002) Innate immunity: the missing link in neuroprotection and neurodegeneration? Nat Rev Neurosci 3:216-227.

Norden DM, Godbout JP (2013) Microglia of the aged brain: primed to be activated and resistant to regulation. Neuropathol Appl Neurobiol 39:1934.

Pickering M, O'Connor JJ (2007) Pro-inflammatory cytokines and their effects in the dentate gyrus. Prog Brain Res 163:339-354.

Pine RW, Wertz MJ, Lennard ES, Dellinger EP, Carrico CJ, Minshew BH (1983) Determinants of organ malfunction or death in patients with intra-abdominal sepsis: a discriminant analysis. Arch Surg 118:242-249.

Prolla TA (2002) DNA microarray analysis of the aging brain. Chem Senses 27:299-306.

Qiao X, Cummins DJ, Paul SM (2001) Neuroinflammation-induced acceleration of amyloid deposition in the APPV717F transgenic mouse. Eur J Neurosci 14:474-482.

Qin L, Wu X, Block ML, Liu Y, Breese GR, Hong JS, Knapp DJ, Crews FT (2007) Systemic LPS causes chronic neuroinflammation and progressive neurodegeneration. Glia 55:453-462.

Semmler A, Frisch C, Debeir T, Ramanathan M, Okulla T, Klockgether T, Heneka MT (2007) Long-term cognitive impairment, neuronal loss and reduced cortical cholinergic innervation after recovery from sepsis in a rodent model. Exp Neurol 204:733-740.

Semmler A, Widmann CN, Okulla T, Urbach H, Kaiser M, Widman G, Mormann F, Weide J, Fliessbach K, Hoeft A, Jessen F, Putensen C, Heneka MT (2013) Persistent cognitive impairment, hippocampal atrophy and EEG changes in sepsis survivors. J Neurol Neurosurg Psychiatry 84:62-69.

Shaw KN, Commins S, O’Mara SM (2001) Lipopolysaccharide causes deficits in spatial learning in the watermaze but not in BDNF expression in the rat dentate gyrus. Behav Brain Res 124:47-54.

Sheng JG, Bora SH, Xu G, Borchelt DR, Price DL, Koliatsos VE (2003) Lipopolysaccharide-induced-neuroinflammation increases intracellular accumulation of amyloid precursor protein and amyloid $\beta$ peptide in APPswe transgenic mice. Neurobiol Dis 14:133-145.

Sparkman NL, Kohman RA, Scott VJ, Boehm GW (2005) Bacterial endotoxin-induced behavioral alterations in two variations of the Morris water maze. Physiol Behav 86:244-251.

Sprung CL, Peduzzi PN, Shatney CH, Schein R, Wilson MF, Sheagren JN, Hinshaw LB (1990) Impact of encephalopathy on mortality in the sepsis syndrome. The Veterans Administration Systemic Sepsis Cooperative Study Group. Crit Care Med 18:801-806.

Strehl A, Lenz M, Itsekson-Hayosh Z, Becker D, Chapman J, Deller T, Maggio N, Vlachos A (2014) Systemic inflammation is associated with a reduction in synaptopodin expression in the mouse hippocampus. Exp Neurol 261:230-235.

Tejera D, Mercan D, Sanchez-Caro JM, Hanan M, Greenberg D, Soreq H, Latz E, Golenbock D, Heneka MT (2019) Systemic inflammation impairs microglial A $\beta$ clearance through NLRP3 inflammasome. EMBO J 38: e101064.

Valero J, Mastrella G, Neiva I, Sánchez S, Malva JO (2014) Long-term effects of an acute and systemic administration of LPS on adult neurogenesis and spatial memory. Front Neurosci 8:83.

Wendeln AC, Degenhardt K, Kaurani L, Gertig M, Ulas T, Jain G, Wagner J, Häsler LM, Wild K, Skodras A, Blank T, Staszewski O, Datta M, Centeno TP, Capece V, Islam MR, Kerimoglu C, Staufenbiel M, Schultze JL, Beyer $\mathrm{M}$, et al. (2018) Innate immune memory in the brain shapes neurological disease hallmarks. Nature 556:332-338.

Widmann CN, Heneka MT (2014) Long-term cerebral consequences of sepsis. Lancet Neurol 13:630-636.

Wilson JX, Young GB (2003) Progress in clinical neurosciences: sepsis-associated encephalopathy: evolving concepts. Can J Neurol Sci 30:98-105. 